\title{
Numerical technique for the 3D microarchitecture design of elastic composites inspired by crystal symmetries
}

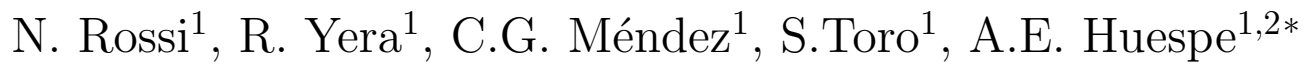 \\ ${ }^{1}$ CIMEC-UNL-CONICET, Predio Conicet "Dr Alberto Cassano", CP 3000 Santa Fe, Argentina \\ ${ }^{2}$ E.T.S d'Enginyers de Camins, Canals i Ports, Technical University of Catalonia (Barcelona Tech) \\ Campus Nord UPC, Mòdul C-1, c/ Jordi Girona 1-3, 08034, Barcelona, Spain
}

Keywords: 3D microarchitecture synthesis; topology optimization algorithm; topology design inspired by crystal symmetries; 3D elastic metamaterials; auxetic materials; pentamode composites; stiffest composite.

\begin{abstract}
A numerical methodology developed for the microarchitecture design of 3D elastic two-phase periodic composites with effective isotropic properties close to the theoretical bounds is here presented and analyzed. This methodology is formulated as a topology optimization problem and is implemented using a level-set approach jointly with topological derivative.

The most salient characteristic of this methodology is the imposition of preestablished crystal symmetries to the designed topologies; we integrate a topological optimization formulation with crystal symmetries to design mechanical metamaterials.

The computational homogenization of the composite elastic properties is determined using a Fast Fourier Transform (FFT) technique. Due to the design domains are the primitive cells of Bravais lattices compatible with the space group imposed to the material layout, we have adapted the FFT technique to compute the effective properties in 3D parallelepiped domains.

In this work, to find the topologies satisfying the proposed targets, we test four space groups of the cubic crystal system. Thus, the achievement of composites with effective elasticity tensor having cubic symmetry is guaranteed, and the isotropic response is then enforced by adding only one scalar constraint to the topology optimization problem.
\end{abstract}

${ }^{*}$ Corresponding author. E-mail address: ahuespe@cimec.unl.edu.ar (A.E. Huespe). 
To assess the methodology, the following microarchitectures are designed and reported: two auxetic composites, three pentamode materials, and one maximum stiffness composite. With only one exception, all the remaining topologies display effective elastic properties with Zener coefficients approximating to 1 .

\section{Introduction}

The microarchitecture synthesis of composites with unusual properties that could be manufactured using a rapid prototyping technique has recently risen an enormous interest in the community, see Kadic et al. [1] and references cited therein. This interest is mainly due to the notorious advances observed in this type of material processing technique that opens the possibility of manufacturing complex microstructures.

Closely related to this topic, the authors of this paper, see Yera et al. [2], have developed a topology design technique for the microarchitecture synthesis of composites where the resulting material configurations at the microscale copy the symmetry of crystals. The design goal is to attain two-phase, stiff and void, composites with periodic microstructures and isotropic effective elastic properties close to the theoretical bounds. The crucial point of this approach is the imposition of crystal symmetries to the designed microarchitectures. This criterion could be adopted as a guiding principle in the design of new microarchitectures. A discussion about the effect that different space groups have on the realization of composites with properties close to the bounds have also been addressed in the contribution of Yera et al. In this work, we study the numerical aspects related to this design methodology.

The above-mentioned topology design technique is formulated as an inverse homogenization problem which closely follows the original methodology reported by Sigmund [3], Sigmund [4] and clearly explained in the book by Bendsoe and Sigmund [5]. In particular, the topology optimization algorithm that we have implemented is based on a level-set-technique with a topological derivative that has been originally reported by Amstutz and Andrä [6] and Amstutz et al. [7]. It is also worth to mention that an analogous methodology, having some elements in common with the here presented procedure, is the level-set technique reported by Allaire and co-authors, see Allaire et al. [8]. Alternative level-set-techniques for topology optimization problems can be found in the review paper by van Dijk et al. [9].

In the present approach, the crystal symmetries are imposed to the attained topologies via a geometrical constraint on the materials configurations that are tested during the topology optimization procedure. Due to the characteristic properties of crystal symmetries, the imposition of these constraints into the topology optimization problem introduces several distinguishing features which can be summarized as follows: i) a correct selection of the space group symmetry will auto- 
matically provide the required symmetry of the target effective physical property. ii) the geometrical symmetry constraining the admissible topologies restricts the design space, or material distribution space, determining a robust performance of the topology optimization algorithm. Furthermore, we show that by imposing suitable crystal symmetries, then, a wide range of topologies can be obtained, including the well-known pentamode diamond-like microarchitecture reported by Milton and Cherkaev [10]. This solution is analyzed in sub-Section 4.2.3.

Intrinsically associated to this design approach is the evaluation of the effective properties of the tested composite through a computational homogenization technique. This step is by far the most demanding computational burden of the optimization algorithm, and we adopt a Fast Fourier Transform (FFT) technique to perform it. In the case of imposing crystal symmetries, the computational homogenization may be conveniently performed in spatial domains with non-conventional shapes, such as suggested by Méndez et al. [11] and Podestá et al. [12]. Due to the FFT technique is not well suited for solving homogenization problems in design domains with complex shapes, we have adapted it to parallelepiped domains, which is sufficient for our purposes. A validation test that we present in this paper shows the performance of the FFT technique to compute the 3D elasticity tensor homogenization of the tested composites.

The authors have analyzed in Yera et al. [2] the use of several space groups of the cubic system to attain two-phase microstructures, one of them is void. In those cases, similar to the present work, the target has been the design of isotropic elastic composites whose effective properties are close to the theoretical bounds of Hashin-Shtrikmann. Here, we specifically present a restricted set of solutions that corresponds to three kind of composites, one auxetic, one maximum stiffness and three pentamodes, using cells with $256 \times 256 \times 256$ voxels. Therefore, these solutions display well-refined details.

A brief description of the paper is the following. First, in Section 2, we summarize the topology design approach followed in this work. We emphasize the description of the FFT technique applied to problems with non-orthogonal domains. Then, this technique is validated, and its performance is assessed and compared with the finite element method.

In Section 3, we particularize the topology design approach for solving microarchitecture design problems of isotropic composites whose effective properties are close to the theoretical bounds. In sub-Section 3.4.1, we address some important issues concerning the implementation of the level-set algorithm and the imposition of crystal symmetries to the tested solutions.

Section 4 presents the numerical assessments of the algorithm. The microarchitectures of some representative composites are designed using six space groups of the cubic crystal system to attain the isotropic response. The conclusions are presented in Section 5. Finally, some specific aspects of the topological derivative of 

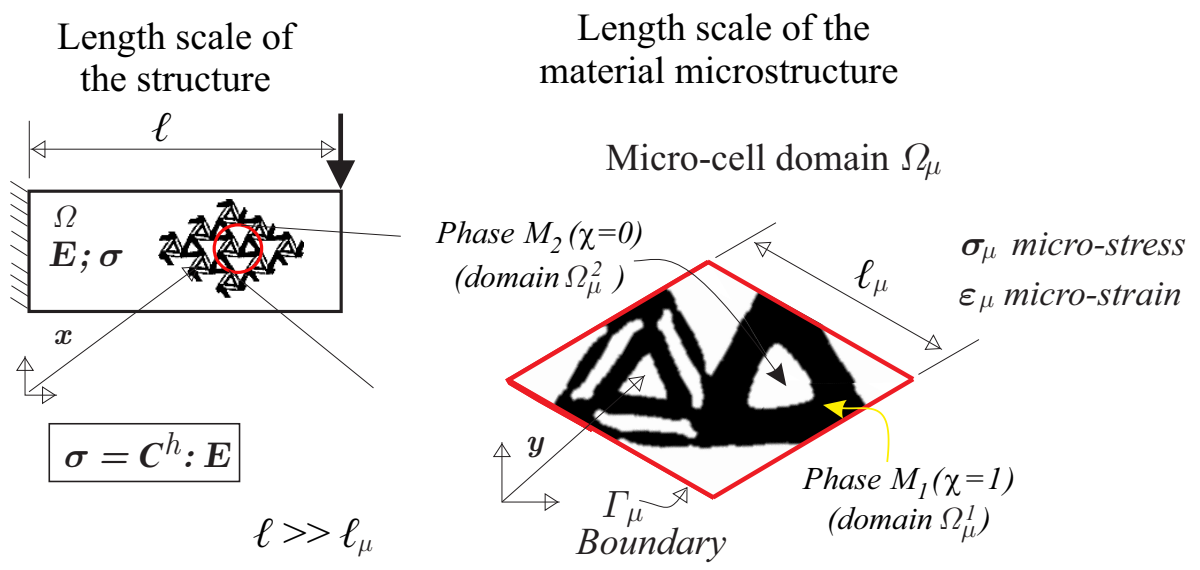

Figure 1: Multiscale structural problem. Cell $\Omega_{\mu}$ of the composite periodic microstructure with phases $M_{1}$ and $M_{2}$. Characteristic function $\chi$. At the macrostructure scale, points are identified with $\boldsymbol{x}$ and the effective elastic properties of the composite are characterized by the homogenized constitutive elasticity tensor $\boldsymbol{C}^{h}$. At the microscale, points are identified with $\boldsymbol{y}$ and elasticity tensors with $\boldsymbol{C}$.

3D homogenization problems, which is a crucial issue of the present approach, are briefly described in Appendix I. The general algorithm is presented in Appendix II.

\section{Topology design approach}

Let us consider the structure sketched in Figure 1 which displays a dominating size $\ell$ and is built with a material composed of two phases $M_{1}, M_{2}$. This composite has a periodic microstructure with a characteristic length, $\ell_{\mu}$. A very fundamental supposition in this work is that $\ell_{\mu} \ll \ell$. It is noteworthy to remark that all periodic microstructures have underlying Bravais lattices, whose features are widely exploited in this work. According to this observation, $\ell_{\mu}$ can be associated with the size of the primitive vectors of the lattice.

At the structural length scale, the effective elastic properties of the composite can be described through the homogenized elasticity tensor $\boldsymbol{C}^{h}$, which relates the macrostrains $\boldsymbol{E}$ with the macro-stresses $\boldsymbol{\sigma}$. A representative cell $\Omega_{\mu}$ of the microstructure is used to compute $\boldsymbol{C}^{h}$. For those composites displaying effective isotropic elastic responses, $\boldsymbol{C}^{h}$ is fully characterized by the effective bulk modulus $\kappa^{h}$ and the effective shear modulus $G^{h}$.

Let us suppose additionally that the material configuration at the microscale can be described with a characteristic function $\chi(\boldsymbol{y})$ defined on $\Omega_{\mu}$, which is 1 in the points $\boldsymbol{y}$ where phase $M_{1}$ exists and is 0 where phase $M_{2}$ exists. Thus, the expressions $\boldsymbol{C}^{h}(\chi), \kappa^{h}(\chi)$ and $G^{h}(\chi)$ manifest that the homogenized elastic properties depend 
on the spatial distribution of phases in $\Omega_{\mu}$.

Under these conditions, a possible topology optimization problem consists of determining the function $\chi$ satisfying the minimum of a given target function, $f\left(\boldsymbol{C}^{h}\right)$ subjected to specified constraints.

A suitable topology optimization problem for the objective pursued in this work is defined in Section 3. But before addressing this topic, we present with some detail, in the next two sub-Sections, two important aspects of the methodology. They are a procedure to select suitable spatial optimization domains, according to the target problem; as well as, an efficient technique for computing the homogenized elasticity tensor $\left(\boldsymbol{C}^{h}\right)$ in such optimization domains. More conventional issues about the formulation and evaluation of the elasticity tensor homogenization are presented in Appendix I.

\subsection{Selection of the design domain $\Omega_{\mu}$ : Bravais lattices and unit cells}

The most salient feature of the present design methodology consists of imposing a predefined crystal symmetry to the designed microarchitecture with the condition that this symmetry is compatible with the target effective elastic symmetry of the problem. For a composite with periodic microstructure, the nexus between the symmetry of its microarchitecture material configuration and the symmetry displayed by its effective physical property is given by the Neumann's principle, broadly applied in crystallography, see Nye [13].

Following this principle, Yera et al. [2] suggest the adoption of a crystal symmetry assuring the fulfillment of the target effective elastic symmetry. Recalling that the target problem in this work aims at attaining the isotropic symmetry, then, in 2D topologies, the hexagonal crystal system guarantees the attainment of this property. Contrarily, there is not any crystal system in 3D problems guaranteeing such symmetry. According to this observation, we will be only testing space groups compatible with the Cubic crystal system because it displays the higher symmetry for 3D topologies.

The geometrical symmetry characterizing a periodic microstructure can be additionally categorized with a specific crystal space group and point group ${ }^{1}$. Every Bravais lattice has a point group symmetry. Therefore, there is a compatibility relationship between the symmetry of the pursued effective property and a given Bravais lattice through the associated point group. This connection has been studied in greater details by the authors in previous works, see [17], [12] and [11].

\footnotetext{
${ }^{1}$ Further details about the classification and properties of space and point groups can be found in [14] and Vainshtein [15]. The readers could also visit the web-page [16] for visualizing the symmetry elements of different space groups.
} 
Using these ideas, we propose a procedure which can be explained with the information described in Table 1. In this Table, we show the compatibility relationships between the space groups, the point groups and the Bravais lattices for the Cubic crystal system.

Let us consider, for example, a target effective property with cubic symmetry. Then, five point groups and 36 space groups are compatible with this symmetry, such as shown in the third and fourth columns of the Table. One of these space groups is chosen with a given criterion, for example, the I23. Automatically, the bcc Bravais lattice is associated with it, such as shown in the last column of the table. Finally, for solving this problem with the imposed symmetry I23, the primitive cell of the bcc Bravais lattice should be tentatively defined as the topology optimization domain $\Omega_{\mu}$. Following the same idea, if the space group $P 23$ were chosen, the simple cubic Bravais lattice is compatible and the corresponding simple cubic primitive cell should be adopted.

\subsection{Computational homogenization using a FFT technique in parallelepipedic domains}

The FFT-based homogenization techniques have been introduced by Moulinec and Suquet [18] for computing the tensor $\boldsymbol{C}^{h}$ of composites with rather arbitrary microstructures. Afterward, these techniques have been improved by several authors, and particularly, Eyre and Milton [19] have introduced a specific algorithm exhibiting better convergence properties for the homogenization of composites with a large stiffness contrast between the two constituent phases. In our implementation, we follow this last approach.

Due to these techniques are now well-established, they are not shown here. Thus, the interested reader is addressed to the reference works for further details of implementations. The only issue which deserves specific attention in this work is the computation of the FFT operations in non-orthogonal domains. The effective elastic properties of the designed composites are computed with micro-cells coinciding with the spatial domains where the topology optimization problems are solved. As explained above, in general, these domains are constituted by parallelepipeds defined by the primitive vectors of Bravais lattices, and therefore, they are not rectangular prisms.

\subsubsection{FFT in non-orthogonal coordinates}

For simplicity, we describe the computation of the FFT in non-orthogonal coordinates using a two-dimensional geometry. Its generalization to $3 \mathrm{D}$ problems is direct. 
Table 1: Cubic System. Compatible point and space groups. Bravais lattices compatible with the cubic systems are: Simple Cubic (SC), Face Centered Cubic (fcc) and Body Centered Cubic (bcc). Primitive cells of the three Bravais lattices with volumes $\left|\Omega_{\mu}^{S C}\right|=1 .,\left|\Omega_{\mu}^{f c c}\right|=0.25$ and $\left|\Omega_{\mu}^{b c c}\right|=0.5$. The elasticity matrix with cubic symmetry in the natural basis is represented in column 1 . The symbol "* - *" linking two coefficients means that they are equal. Only three coefficients define the elasticity matrix in natural basis. Point and spatial groups denoted in bold text are tested in this work. The number of symmetry elements in space and point groups increases toward the descending direction of rows.

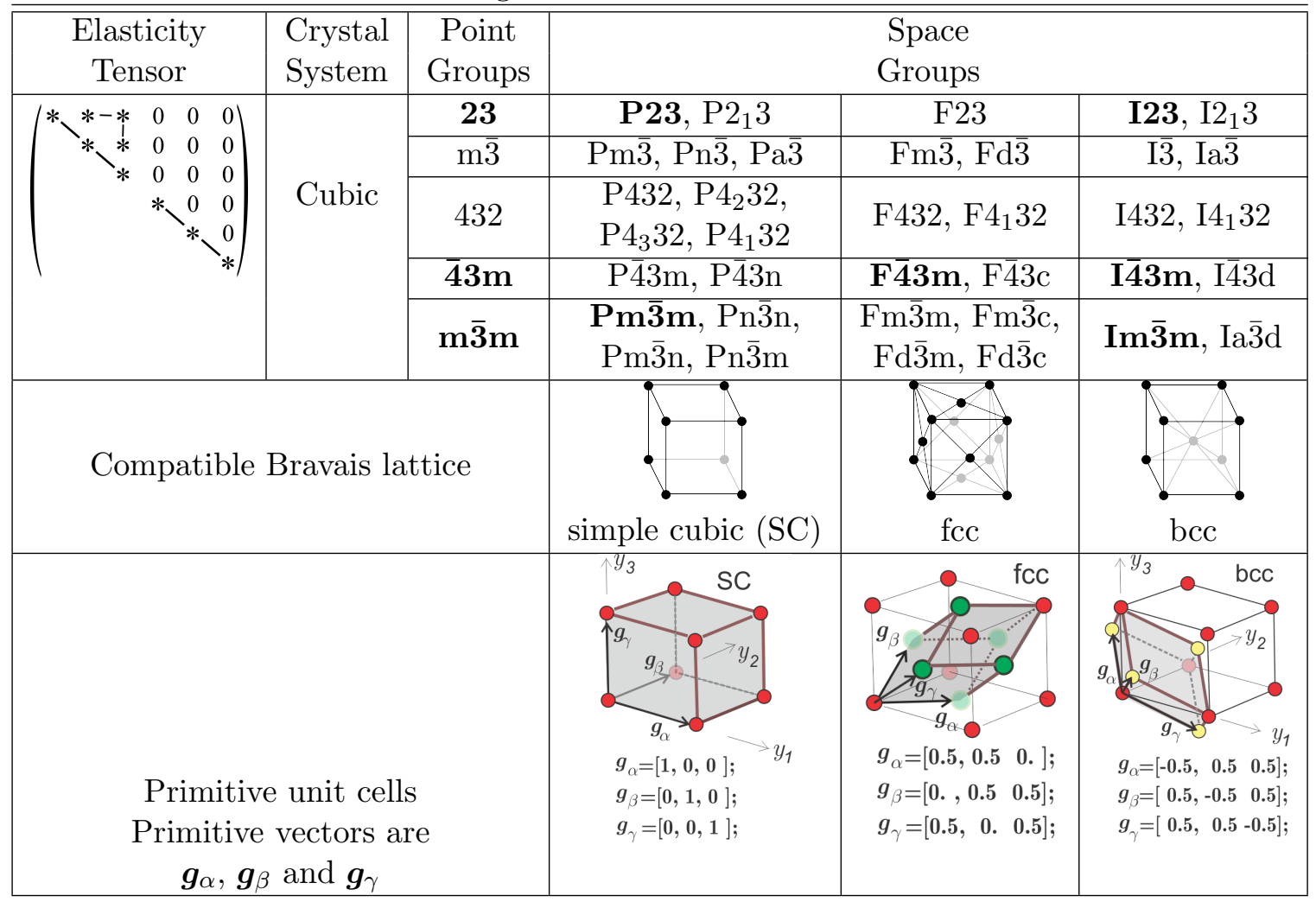


Let us consider a periodic material whose underlying Bravais lattice has nonorthogonal primitive vectors, $\boldsymbol{g}_{\alpha}$ and $\boldsymbol{g}_{\beta}$, such as sketched in Figure 2. We introduce the basis $\left\{\boldsymbol{g}_{\alpha}, \boldsymbol{g}_{\beta}\right\}$ to define a non-orthogonal coordinate system with an homogeneous metric tensor. A position vector $\boldsymbol{y}$ in this basis is expressed according to $\boldsymbol{y}=\theta^{\alpha} \boldsymbol{g}_{\alpha}+\theta^{\beta} \boldsymbol{g}_{\beta}$ ( $\alpha$ and $\beta$ identify different basis vectors, and therefore, the summation convention of repeated index is not applied), where $\left\{\theta^{\alpha}, \theta^{\beta}\right\}$ are the corresponding pair of coordinates, i.e., the contravariant components of $\boldsymbol{y}$.
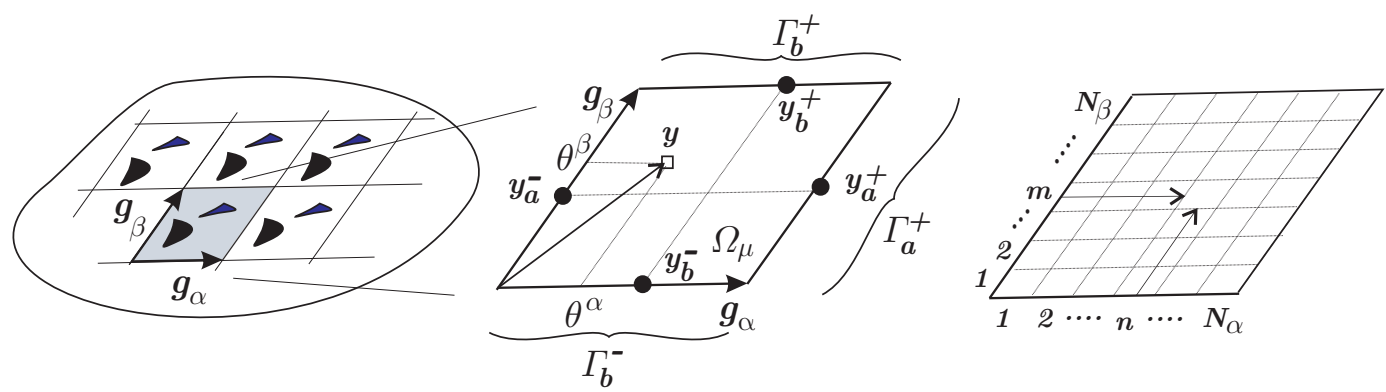

Figure 2: Periodic microcell along non-orhogonal directions $\boldsymbol{g}_{\alpha}$ and $\boldsymbol{g}_{\beta}$. Identification of the $n m$-th pixel.

Let us also consider a periodic mechanical problem such that the fluctuation of the micro-displacements $\tilde{\boldsymbol{u}}$ and micro-tractions $\boldsymbol{t}$ are periodic and antiperiodic, respectively, along the directions $\boldsymbol{g}_{\alpha}$ and $\boldsymbol{g}_{\beta}$. Therefore, by defining the unit cell domain $\Omega_{\mu}$, the following identities are satisfied on its boundary:

$$
\begin{array}{rr}
\tilde{\boldsymbol{u}}\left(\boldsymbol{y}_{a}^{+}\right)=\tilde{\boldsymbol{u}}\left(\boldsymbol{y}_{a}^{-}\right) ; \boldsymbol{t}\left(\boldsymbol{y}_{a}^{+}\right)=-\boldsymbol{t}\left(\boldsymbol{y}_{a}^{-}\right) \quad \forall \boldsymbol{y}_{a}^{+} \in \Gamma_{a}^{+} \text {and } \boldsymbol{y}_{a}^{+}=\boldsymbol{y}_{a}^{-}+\boldsymbol{g}_{\alpha} \\
\tilde{\boldsymbol{u}}\left(\boldsymbol{y}_{b}^{+}\right)=\tilde{\boldsymbol{u}}\left(\boldsymbol{y}_{b}^{-}\right) ; \boldsymbol{t}\left(\boldsymbol{y}_{b}^{+}\right)=-\boldsymbol{t}\left(\boldsymbol{y}_{b}^{-}\right) \quad \forall \boldsymbol{y}_{b}^{+} \in \Gamma_{b}^{+} \text {and } \boldsymbol{y}_{b}^{+}=\boldsymbol{y}_{b}^{-}+\boldsymbol{g}_{\beta}
\end{array}
$$

Furthermore, we can express every component of the displacements, the strains and the stresses as fields in terms of the contravariant coordinates. Let one of these periodic function, for example a strain component, be denoted $f\left(\theta^{\alpha}, \theta^{\beta}\right)$. Then, $f$ satisfies $f\left(\theta^{\alpha}+\Delta \theta^{\alpha}, \theta^{\beta}\right)=f\left(\theta^{\alpha}, \theta^{\beta}\right)$ and $f\left(\theta^{\alpha}, \theta^{\beta}+\Delta \theta^{\beta}\right)=f\left(\theta^{\alpha}, \theta^{\beta}\right)$ with $\Delta \theta^{\alpha}=1$ and $\Delta \theta^{\beta}=1$. These fields can be transformed to the frequency domain using the Fourier Transform along the directions $\boldsymbol{g}_{\alpha}$ and $\boldsymbol{g}_{\beta}$ :

$$
\hat{f}\left(\zeta_{\alpha}, \zeta_{\beta}\right)=\int_{0}^{1} \int_{0}^{1} f\left(\theta^{\alpha}, \theta^{\beta}\right) e^{-i\left(\zeta_{\alpha} \theta^{\alpha}+\zeta_{\beta} \theta^{\beta}\right)} d \theta^{\alpha} d \theta^{\beta} ;
$$

where the two frequencies $\left(\zeta_{\alpha}, \zeta_{\beta}\right)$ can be interpreted as the covariant components of the frequency vector $\boldsymbol{\xi}=\zeta_{\alpha} \boldsymbol{G}^{\alpha}+\zeta_{\beta} \boldsymbol{G}^{\beta}$, being $\left\{\boldsymbol{G}^{\alpha}, \boldsymbol{G}^{\beta}\right\}$ the reciprocal basis of $\left\{\boldsymbol{g}_{\alpha}, \boldsymbol{g}_{\beta}\right\}$ satisfying $\boldsymbol{G}^{i} \cdot \boldsymbol{g}_{j}=\delta_{i j}$ with $\delta_{i j}$ the Kronecker delta function.

By taking into account these preliminary concepts, the homogenized properties of periodic composites characterized by unit cells defined by non-orthogonal primitive 
vectors can be computed with the FFT algorithm. A brief summary of this algorithm implementation is next described:

1 First, the unit cell is partitioned in $\left(N_{\alpha} \times N_{\beta}\right)$ pixels ${ }^{2} ; N_{\alpha}$ in the direction $\boldsymbol{g}_{\alpha}$ and $N_{\beta}$ in the direction $\boldsymbol{g}_{\beta}$, such as shown in Figure 2. The following set of discrete frequencies are defined:

$$
\begin{array}{r}
\boldsymbol{\zeta}_{\alpha}=\left[\zeta_{\alpha}^{(1)}, \zeta_{\alpha}^{(2)}, \ldots, \zeta_{\alpha}^{(n)}, \ldots, \zeta_{\alpha}^{\left(N_{\alpha}\right)}\right]=\left[-\frac{N_{\alpha}}{2}+1,-\frac{N_{\alpha}}{2}+2, \ldots, \frac{N_{\alpha}}{2}\right] ; \\
\boldsymbol{\zeta}_{\beta}=\left[\zeta_{\beta}^{(1)}, \zeta_{\beta}^{(2)}, \ldots, \zeta_{\beta}^{(m)}, \ldots, \zeta_{\beta}^{\left(N_{\beta}\right)}\right]=\left[-\frac{N_{\beta}}{2}+1,-\frac{N_{\beta}}{2}+2, \ldots, \frac{N_{\beta}}{2}\right] ; \\
\quad \text { for } N_{\alpha} \text { and } N_{\beta} \text { even }
\end{array}
$$

(if $N_{\alpha}$ or $N_{\beta}$ are odd, the formula in Moulinec et al. can be used). From them, we define the $\left(N_{\alpha} \times N_{\beta}\right)$ vectors of discrete frequencies transformed to the Cartesian coordinates:

$$
\left[\boldsymbol{\xi}^{(n, m)}\right]=\zeta_{\alpha}^{(n)} \boldsymbol{G}^{\alpha}+\zeta_{\beta}^{(m)} \boldsymbol{G}^{\beta}
$$

2 The Green operator in the frequency space, $\boldsymbol{\Gamma}^{0}$, is evaluated for every vector $\boldsymbol{\xi}^{(n, m)}$ as follows:

$$
\Gamma^{0}\left(\boldsymbol{\xi}^{(n, m)}\right)=\boldsymbol{\xi}^{(n, m)}\left(\boldsymbol{\xi}^{(n, m)} \cdot \boldsymbol{C}^{0} \cdot \boldsymbol{\xi}^{(n, m)}\right)^{-1} \boldsymbol{\xi}^{(n, m)}
$$

with $\boldsymbol{C}^{0}$ being a reference elasticity tensor. Note that equation (4), expressed in intrinsic notation, could be computed using Cartesian components, in particular $\boldsymbol{C}^{0}$ is the conventional matrix of the elastic Hooke law in Cartesian components.

3 Proceed in a standard way with the algorithm defined in Eyre et al.

Note that the stress and strain fields in both, space and frequency, domains that arise in the algorithm (10) of the reference work have the components described in Cartesian basis.

In the following, we use indices with Arabic numerals for denoting the components of tensors in Cartesian coordinates. Additionally, we use Kelvin notation for second and fourth order tensors. The stress tensor components $\left\{\sigma_{y_{1} y_{1}}, \sigma_{y_{2} y_{2}}, \sigma_{y_{3} y_{3}}, \sigma_{y_{1} y_{2}}, \sigma_{y_{1} y_{3}}, \sigma_{y_{2} y_{3}}\right\}$ are represented with Arabic numeral indices as follows: $\left\{\sigma_{1}, \sigma_{2}, \sigma_{3}, \sigma_{4}, \sigma_{5}, \sigma_{6}\right\}$, respectively. The stress vector in Kelvin notation results $\left(\sigma_{1}, \sigma_{2}, \sigma_{3}, \sqrt{2} \sigma_{4}, \sqrt{2} \sigma_{5}, \sqrt{2} \sigma_{6}\right)$. An identical criterion is used for strains. In consequence, the corresponding elasticity matrix are denoted with two Arabic indices: $C_{i j}$, with $i, j=1, \ldots, 6$.

\footnotetext{
${ }^{2}$ The generalization to three-dimensional microcell homogenization is direct by considering $N_{\alpha} \times$ $N_{\beta} \times N_{\gamma}$ voxels.
} 


\subsubsection{Validation tests}

Next, we validate the FFT technique. The effective elasticity tensor of a composite constituted of a stiff phase with an array of spherical voids geometrically placed in the positions occupying the atoms of a face-centered cubic (fcc) Bravais lattice is computed. Then, we analyze the computational cost (time) vs. accuracy that demands to solve this problem with the FFT, using different densities of grids. We also compare these results with those obtained with the Finite Element Method (FEM).

Due to the symmetry of this microarchitecture, the effective elasticity tensor displays cubic symmetry. The Young modulus of the stiff phase, $M_{1}$, is $E_{1}=1$ and the Poisson's ratio is $\nu_{1}=0.4$. The spherical voids have a radius of 0.15 , wherewith the volume fraction of the composite stiff phase is $f_{1}=0.9435$. The analytic reference solution of the effective elastic moduli, reported by Cohen [20], is given by: $\left(C_{r e f}^{h}\right)_{11}=\left(C_{\text {ref }}^{h}\right)_{22}=\left(C_{r e f}^{h}\right)_{33}=1.7415,\left(C_{r e f}^{h}\right)_{12}=\left(C_{r e f}^{h}\right)_{23}=\left(C_{r e f}^{h}\right)_{13}=$ 1.0982 and $\left(C_{r e f}^{h}\right)_{44}=\left(C_{r e f}^{h}\right)_{55}=\left(C_{r e f}^{h}\right)_{66}=0.6456$. This solution is calculated considering that the second phase is void.

In the numerical model, the void phase, $M_{2}$, is assumed to be a soft elastic material whose Young modulus is $E_{2}=\gamma E_{1}$ with the contrast factor $\gamma=10^{-4}$. We also show here that this factor is small enough to simulate the void. The Poisson's ratio is taken as $\nu_{2}=\nu_{1}$.

The computational homogenization is evaluated on a primitive micro-cell $\Omega_{\mu}^{p}$ of the fcc lattice, such as displayed in Figure 3-c. Conventional and primitive cells are shown in Figure 3-a and c. Superscript $p$ and $c$ indicates primitive and conventional unit cells, respectively. The conventional unit cell size is $1 \times 1 \times 1$. Note that the primitive cell has a volume $\left|\Omega_{\mu}^{p}\right|=0.25$ compared with the volume of the conventional unit cell whose volume is $\left|\Omega_{\mu}^{c}\right|=1$. Therefore, to attain similar accuracies, the micro-cell $\Omega_{\mu}^{c}$ requires 4 times more voxels than the micro-cell $\Omega_{\mu}^{p}$. This feature of the primitive cell is remarkably most important for the design of microarchitectures. In fact, for the same composite, the primitive cell displays simpler topologies respect to those described by the conventional unit cell.

Results are plotted in Figure 4. The curves show the computation time needed for solving every problem vs. the solution error using the primitive cell $\Omega_{\mu}^{p}$. The errors are computed as the normalized Frobenius norm of the difference between the computed elasticity tensor $\boldsymbol{C}^{h}$ and the reference one $\boldsymbol{C}_{r e f}^{h}$. The numbers in the Figure indicate the grid densities and represent the number of voxels in each dimension. For example, the number 300 indicates that the solution is obtained with a discretization of $300 \times 300 \times 300$ voxels. The finite element mesh is built by taking the same spatial distribution of voxels and dividing each one of them into six linear tetrahedra. Solutions denoted "without greys" are obtained with voxels (FFT) or finite elements (FEM) having the properties of only one of the two phases. 


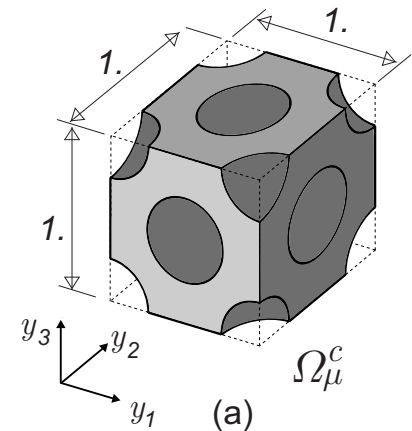

(a)
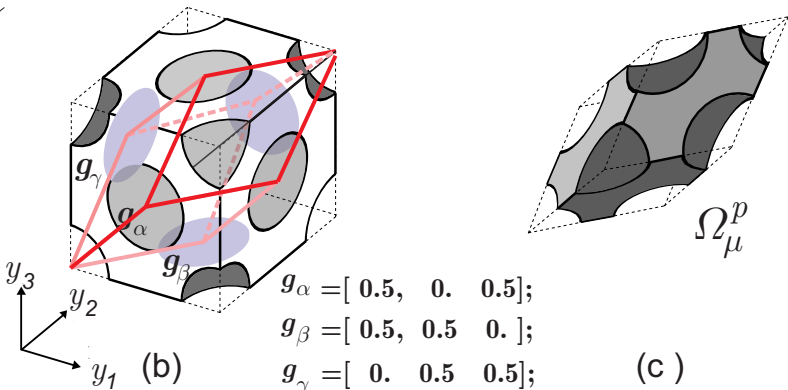

(c)

Figure 3: Validation tests. Composite microstructure. (a) Conventional unit cell $\Omega_{\mu}^{c}$; (b-c) primitive unit cell $\Omega_{\mu}^{p}$. The primitive vectors $\boldsymbol{g}_{\alpha}, \boldsymbol{g}_{\beta}$ and $\boldsymbol{g}_{\gamma}$ also define the periodicity directions.

On the other hand, solutions denoted "with greys" apply the below formulas (20) and (21) to define the material properties of voxels and tetrahedra. In the case of the FFT solutions, the eight vertices of each voxel are used for this determination, but for the FEM solutions, the four nodes of every tetrahedron are used to define the property of the finite element.

As expected, the FEM and FFT methodologies tend to the correct solution by increasing the grid density. However, it is notable the effect induced by the inclusion of the grey material in both methodologies. This effect is still more remarkably in the FFT technique, there are striking differences in favor of this technique. To attain similar error levels in the computation of the effective elasticity tensor, the cost of FEM solutions is more than one order higher than that using the FFT technique. A different conclusion would be attained if grey elements were removed from the model.

The FEM uses a linear equation system iterative solver (conjugate gradient method) with incomplete Cholesky preconditioning (IChol). The error tolerances for both methodologies (FEM with iterative solver and FFT) have been fixed to $10^{-6}$, and their implementations have been optimized to run in MATLAB using an Intel i9-9900K Processor with 64GB of memory ram and Intel Turbo Boost activated.

\section{Microarchitecture design of isotropic elastic com- posites}

\subsection{Bounds of the effective elastic property of two-phase well-ordered composites}

Let us consider the full set of periodic composites with isotropic effective elastic properties and two isotropic elastic phases $M_{1}$ and $M_{2}$ whose bulk moduli are $\kappa_{1}$, 


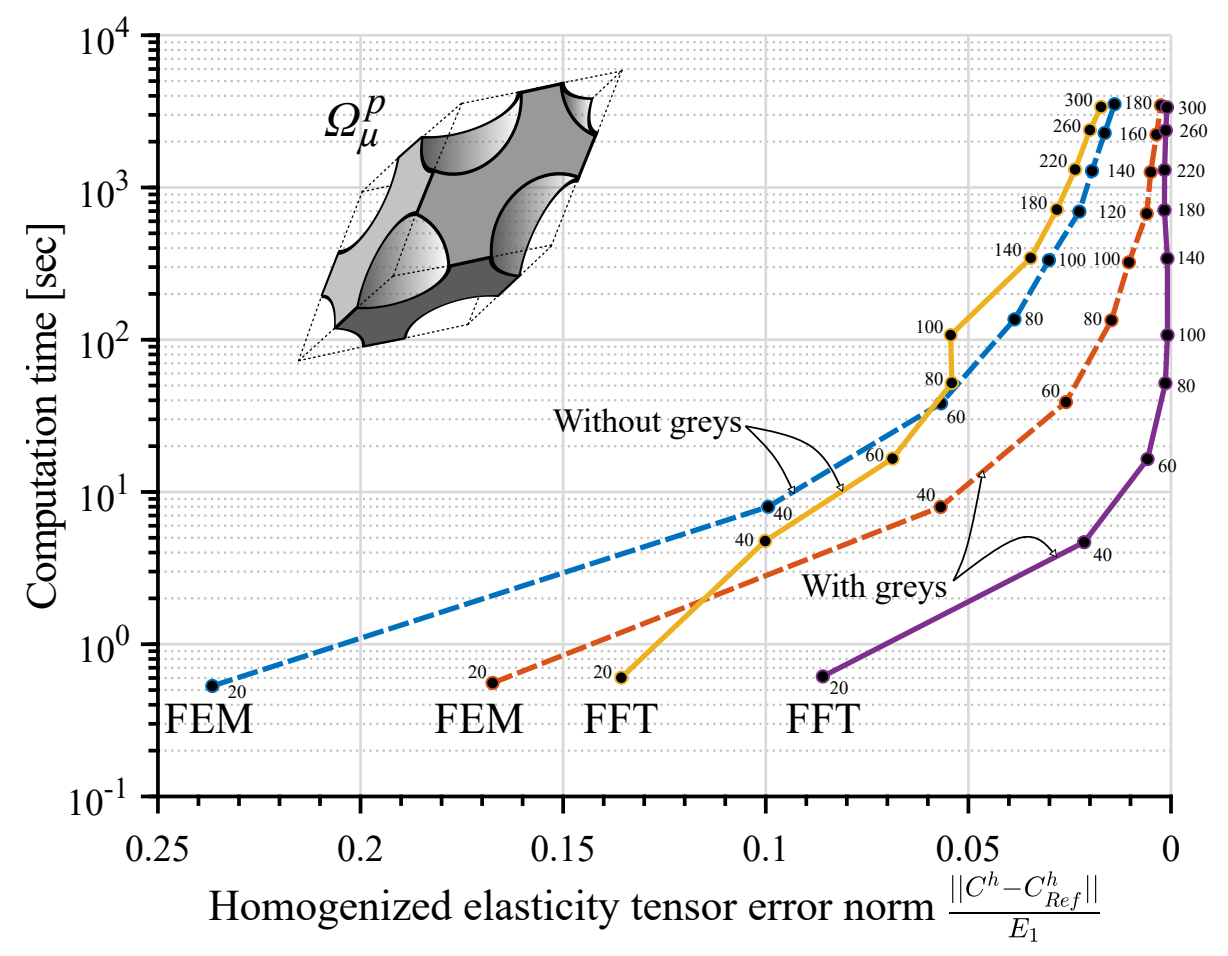

Figure 4: Computation time vs. normalized errors to evaluate the homogenized elasticity tensors (Frobenius norm). Solutions obtained with a primitive cell $\left(\Omega_{\mu}^{p}\right)$. Numbers indicate the grid density size of each solution. Comparison of FEM vs. FFT solutions. 


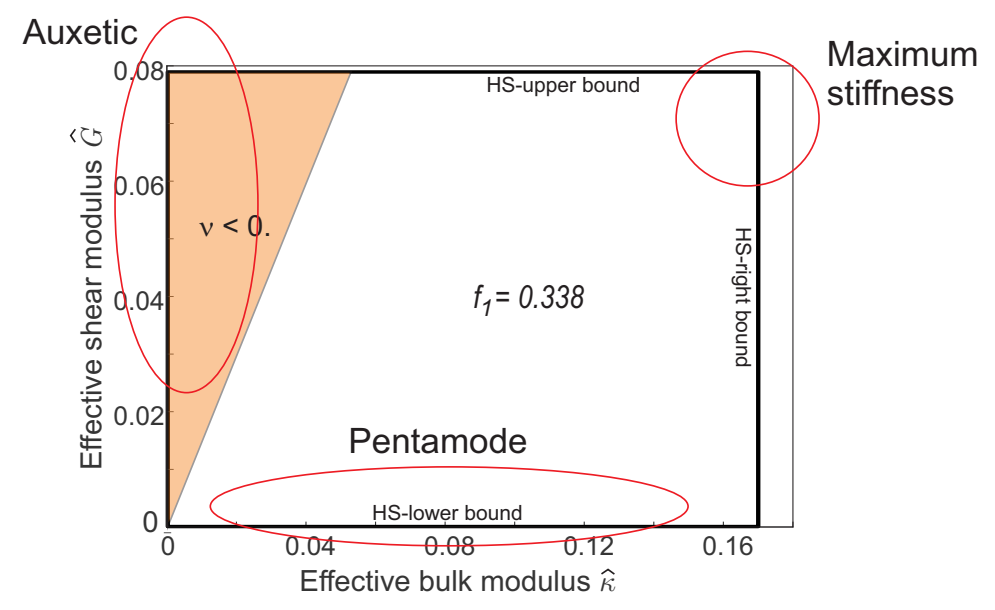

Figure 5: Theoretical bounds of effective properties for two-phase isotropic 3D composites according to Hashin and Shtrikman [22] (HS-bounds). Properties of phases $M_{1}$ and $M_{2}$ are $\kappa_{1}=1.667, G_{1}=0.3571, \kappa_{2}=\gamma \kappa_{1}, G_{2}=\gamma G_{1}$, contrast factor $\gamma=10^{-4}$, volume fraction $f_{1}=0.338$.

$\kappa_{2}$, with $\kappa_{2}<\kappa_{1}$, and shear moduli $G_{1}, G_{2}$, with $G_{2}<G_{1}$, respectively, and a volume fraction of $M_{1}$ equal to $f_{1}$. Then, the effective properties of these composites, typically the bulk and shear effective moduli, $\hat{\kappa}$ and $\hat{G}$, are bounded: $\hat{\kappa}^{l} \leq \hat{\kappa} \leq \hat{\kappa}^{u}$, $\hat{G}^{l} \leq \hat{G} \leq \hat{G}^{u}$, where $\hat{\kappa}^{u}$ and $\hat{\kappa}^{l}$ are the upper and lower bounds of the effective bulk modulus, and, $\hat{G}^{u}$ and $\hat{G}^{l}$ are the upper and lower bounds of the effective shear modulus. These bounds have been theoretically estimated with the expressions derived by ${ }^{3}$ Hashin and Shtrikman [22] as follows:

$$
\begin{gathered}
\hat{\kappa}^{u}=\kappa_{1}+\frac{1-f_{1}}{\frac{1}{\kappa_{2}-\kappa_{1}}+3 \frac{f_{1}}{3 \kappa_{1}+4 G_{1}}} \quad ; \quad \hat{\kappa}^{l}=\kappa_{2}+\frac{f_{1}}{\frac{1}{\kappa_{1}-\kappa_{2}}+3 \frac{1-f_{1}}{3 \kappa_{2}+4 G_{2}}} ; \\
\hat{G}^{u}=G_{1}+\frac{1-f_{1}}{\frac{1}{G_{2}-G_{1}}+\frac{6\left(\kappa_{1}+2 G_{1}\right) f_{1}}{5 G_{1}\left(3 \kappa_{1}+4 G_{1}\right)}} \quad ; \quad \hat{G}^{l}=G_{2}+\frac{f_{1}}{\frac{1}{G_{1}-G_{2}}+\frac{6\left(\kappa_{2}+2 G_{2}\right)\left(1-f_{1}\right)}{5 G_{2}\left(3 \kappa_{2}+4 G_{2}\right)}} .
\end{gathered}
$$

For composites constituted of two phases characterized by $\kappa_{1}=1.667, G_{1}=0.3571$, $\kappa_{2}=\gamma \kappa_{1}, G_{2}=\gamma G_{1}$, with the contrast factor $\gamma=10^{-4}$, and a volume fraction $f_{1}=$ 0.338 , these bounds are: $\hat{\kappa}^{u}=0.170 ; \hat{\kappa}^{l}=2.8 \times 10^{-4} ; \hat{G}^{u}=0.789 ; \hat{G}^{l}=7.3 \times 10^{-5}$ and they are plotted in Figure 5 and are denoted the HS-bounds.

\subsection{Topology optimization problems}

The goal is to design the family of two-phase composites with a predefined volume fraction, whose effective properties are isotropic and furthermore, they are the closest

\footnotetext{
${ }^{3}$ Tighter bounds were reported by Berryman and Milton [21]. However, the bounds coincide with the Hashin-Shtrikmann ones when one of the phases is void.
} 
one to the four HS-bounds. The mathematical formulations chosen for solving this problem are the following.

Given the micro-cell $\Omega_{\mu}$, we define the characteristic function $\chi(\boldsymbol{y})$ identifying the positions where the phase $M_{1}$ is placed. It is defined by:

$$
\chi(\boldsymbol{y})= \begin{cases}0 & \forall \boldsymbol{y} \in \Omega_{\mu}^{2} \\ 1 & \forall \boldsymbol{y} \in \Omega_{\mu}^{1}\end{cases}
$$

Then,

i) To approach the inferior HS-bound, the problem is written as:

$$
\begin{array}{cl}
\min _{\chi} G^{h}(\chi) & \\
\text { such that: } \quad & \kappa^{h}(\chi)-\kappa^{t g}=0 \\
& C^{h}(\chi) \text { is isotropic } \\
& f_{1}^{h}(\chi)-f_{1}^{t g}=0
\end{array}
$$

where $\kappa^{t g}$ is the target bulk modulus of the composite defined within the interval $\hat{\kappa}^{l} \leq \kappa^{t g} \leq \hat{\kappa}^{u}, f_{1}^{h}(\chi)=\int_{\Omega_{\mu}} \chi d \Omega$ is the volume fraction of phase $M_{1}$ and $f_{1}{ }^{t g}$ is the target volume fraction. The solutions approaching the HS-lower bound can be obtained by solving a series of problems (7), with $\kappa^{t g}$ sweeping the range of the interval $\left[\hat{\kappa}^{l}, \hat{\kappa}^{u}\right]$.

ii) An alternative problem is formulated to approach the left Hashin-Strikman bound, as follows:

$$
\min _{\chi} \kappa^{h}(\chi)
$$

such that: $\quad G^{h}(\chi)-G^{t g}=0$

$$
\begin{aligned}
& C^{h}(\chi) \text { is isotropic } \\
& f_{1}^{h}(\chi)-f_{1}{ }^{t g}=0
\end{aligned}
$$

where now, the target effective shear modulus $G^{t g}$ is chosen from the interval $\hat{G}^{l} \leq G^{t g} \leq \hat{G}^{u}$. Similarly as described above, the solutions approaching the HS-left bound can be obtained by solving a series of problems with $G^{t g}$ sweeping the range of the interval $\left[\hat{G}^{l}, \hat{G}^{u}\right]$.

iii) In a similar way, the Hashin-Strikman superior and right bounds are approached by changing the minimization problems in (7) and (8) respectively, by one of maximization. 


\subsection{Rephrasing the topology optimization problems for mi- crostructures with cubic crystal symmetries}

Microstructures possessing a cubic crystal system have effective elastic responses with cubic symmetry. Thus, such as shown in Table 1, the coefficients of their elasticity matrices satisfy the following identities: $C_{11}^{h}=C_{22}^{h}=C_{33}^{h} ; C_{12}^{h}=C_{13}^{h}=$ $C_{23}^{h} ; C_{44}^{h}=C_{55}^{h}=C_{66}^{h}$. The remaining coefficients are strictly equal to zero.

According to this observation, as long as it can be guaranteed in advance that the microstructure will have a cubic system, the topology optimization problems (7)-(8) can be rephrased in terms of the elasticity matrix coefficients as follows:

$$
\begin{aligned}
\min _{\psi \in C^{0}} C_{44}^{h} ; & \\
\text { such that: } \quad h_{1} & =C_{11}^{h}+2 C_{12}^{h}-3 \kappa^{t g}=0 \\
h_{2} & =C_{11}^{h}-C_{12}^{h}-C_{44}^{h}=0 \\
h_{3} & =f_{1}^{h}(\chi)-f_{1}^{t g}=0 \\
\min _{\psi \in C^{0}}\left(C_{12}^{h}+\frac{1}{3} C_{44}^{h}\right) ; & \\
\operatorname{such}_{1} \text { that: } \quad & \\
h_{1} & =C_{11}^{h}-C_{12}^{h}+\frac{3}{2} C_{44}^{h}-5 G^{t g}=0 \\
h_{2} & =C_{11}^{h}-C_{12}^{h}-C_{44}^{h}=0 \\
h_{3} & =f_{1}^{h}(\chi)-f_{1}^{t g}=0
\end{aligned}
$$

Note that the coefficients of any isotropic elasticity tensor satisfy the following conditions: a) $C_{44}^{h}=2 G$, b) $C_{12}^{h}+\frac{1}{3} C_{44}^{h}=\kappa$, c) $C_{11}+2 C_{12}=3 \kappa$, and d) $C_{11}-C_{12}+$ $3 C_{44} / 2=5 G$; being $\kappa$ and $G$ the bulk and shear moduli of the material. Thus, after assuming that $\kappa$ is the target bulk modulus of the problem (7), the objective function of this problem is replaced by the identity $a$ ). The first constraint can be replaced by $c$ ), which results the constraint $h_{1}$ in the rephrased formulation (9), while $h_{2}$ of the rephrased formulation ensures the isotropic response. An identical consideration can be applied to reformulate the problem (8). In this case, by assuming that $G$ is the target shear modulus, the objective function of (8) is replaced by $b$ ), the first constraint is replaced by $d$ ), which results the constraints $h_{1}$ in the rephrased formulation (10) and constraint $h_{2}$ ensures the isotropic response.

\subsection{Level-Set topology optimization algorithm}

The topology optimization algorithm for solving the problems (9)-(10) uses the level-set method, combined with topological derivatives. The topological derivative provides the sensitivity of the target properties with changes of the characteristic 
function $\chi$. The numerical technique based on these ingredients, and which is used in this paper, follows the original ideas of the algorithm presented by Amstutz and Andrä [6] and Amstutz et al. [7]. The topological derivative concept is well described in the book by Novotny and Sokołowski [23]. Alternative formulations of topology optimizations problems using topological derivative and level-set functions have also been reported in the works of Allaire and co-authors, see Allaire et al. [8] and references cited therein.

Let us introduce a smooth level-set function $\psi$ defined in the microcell $\Omega_{\mu}, \psi \in$ $C^{0}\left(\Omega_{\mu}\right)$, satisfying

$$
\psi(\boldsymbol{y})=\left\{\begin{array}{cc}
>0 & \forall \boldsymbol{y} \in \Omega_{\mu}^{2} \\
<0 & \forall \boldsymbol{y} \in \Omega_{\mu}^{1} \\
=0 & \forall \boldsymbol{y} \in \partial \Omega_{\mu}^{1}
\end{array},\right.
$$

then, the characteristic functions $\chi(\boldsymbol{y})$ in $\Omega_{\mu}$, given by expression (6), can be redefined as follows:

$$
\chi_{\psi}=\left\{\begin{array}{ll}
1 & \forall \psi \leq 0 \\
0 & \forall \psi>0
\end{array} .\right.
$$

and problems (9)-(10) can be written in terms of $\chi_{\psi}$ :

$$
\begin{aligned}
& \min _{\psi \in C^{0}} C_{44}^{h}\left(\chi_{\psi}\right) \text {; } \\
& \text { such that: } \quad h_{1}=C_{11}^{h}\left(\chi_{\psi}\right)+2 C_{12}^{h}\left(\chi_{\psi}\right)-3 \kappa^{t g}=0 \\
& h_{2}=C_{11}^{h}\left(\chi_{\psi}\right)-C_{12}^{h}\left(\chi_{\psi}\right)-C_{44}^{h}\left(\chi_{\psi}\right)=0 \\
& h_{3}=f_{1}^{h}\left(\chi_{\psi}\right)-f_{1}^{t g}=0 \\
& \min _{\psi \in C^{0}}\left(C_{12}^{h}\left(\chi_{\psi}\right)+\frac{1}{3} C_{44}^{h}\left(\chi_{\psi}\right)\right) \\
& \text { such that: } \quad h_{1}=C_{11}^{h}\left(\chi_{\psi}\right)-C_{12}^{h}\left(\chi_{\psi}\right)+\frac{3}{2} C_{44}^{h}\left(\chi_{\psi}\right)-5 G^{t g}=0 \\
& h_{2}=C_{11}^{h}\left(\chi_{\psi}\right)-C_{12}^{h}\left(\chi_{\psi}\right)-C_{44}^{h}\left(\chi_{\psi}\right)=0 \\
& h_{3}=f_{1}^{h}\left(\chi_{\psi}\right)-f_{1}^{t g}=0
\end{aligned}
$$

By making use of an augmented Lagrangian technique, see Lopes et al. [24], problem (13) (similarly for (14)) is reformulated as follows:

$$
\max _{\lambda} \min _{\psi} \mathcal{T}\left(\chi_{\psi}, \lambda\right)
$$

with:

$$
\mathcal{T}\left(\chi_{\psi}, \lambda\right)=C_{44}^{h}+\lambda_{1} h_{1}+\lambda_{2} h_{2}+\lambda_{3} h_{3}+\frac{\alpha}{2}\left(h_{1}^{2}+h_{2}^{2}+h_{3}^{2}\right)
$$

where the $\lambda_{i}$, with $i=1,2,3$, are the Lagrange multipliers and $\alpha$ is the penalty parameter of the augmented term. 
The solution of problem (15) is found by using a descent direction algorithm. Neither the Lagrangian (16) nor the fields involved in this expression are differentiable for changes of topologies. However, the sensitivities of the field and the Lagrangian can be obtained by applying the topological derivative concept which formally follows the rules of the differential calculus, typically, the chain rule, such as suggested by Amstutz and Andrä [6].

Following the above-mentioned reference works on this topic, the topological derivative of $\mathcal{T}$ at point $\boldsymbol{y}$, denoted $D_{T} \mathcal{T}(\boldsymbol{y})$, provides the sensitivity of the functional $\mathcal{T}$ with changes of $\chi$ at the same point. Let us consider the perturbed characteristic function, $\chi_{\epsilon}$, of the characteristic function $\chi$, that is defined after an interchange of phases within a ball of radius $\epsilon$ and centered at the point $\boldsymbol{y}$. Then the topological derivative satisfies:

$$
\mathcal{T}\left(\chi_{\epsilon}, \lambda\right)=\mathcal{T}(\chi, \lambda)+f(\epsilon) D_{T} \mathcal{T}(\boldsymbol{y}, \lambda)+\mathcal{O}\left(\epsilon^{3}\right)
$$

where $f(\epsilon)$ is a term proportional to the perturbed ball volume, and therefore, to $\epsilon^{3}$ in $3 \mathrm{D}$ problems. Equation (17) allows to identify $D_{T} \mathcal{T}(\boldsymbol{y}, \lambda)$ as the element indicating, at every point of the domain, if a phase change would induce, or not, a decrease of the functional $\mathcal{T}$. This criteria naturally provides a descent direction for modifying the level-set function during an iterative process. This point is further developed in sub-Section 3.4.1.

The expression for the topological derivative of $\mathcal{T}$ can be written in terms of the topological derivative of the components of the homogenized elasticity tensor $C_{i j}^{h}$, denoted $D_{T} C_{i j}^{h}$, by simply applying the conventional chain rule to (16), as follows:

$$
D_{T} \mathcal{T}(\boldsymbol{y}, \lambda)=D_{T} C_{44}^{h}(\boldsymbol{y})+\sum_{i=1}^{3} \lambda_{i} D_{T} h_{i}(\boldsymbol{y})+\alpha \sum_{i=1}^{3}\left(h_{i} D_{T} h_{i}(\boldsymbol{y})\right)
$$

and the expressions of the derivatives of the constrains $D_{T} h_{i}$ are:

$$
\begin{aligned}
& D_{T} h_{1}=D_{T} C_{11}^{h}+2 D_{T} C_{12}^{h} \\
& D_{T} h_{2}=D_{T} C_{11}^{h}-D_{T} C_{12}^{h}-D_{T} C_{44}^{h} \\
& D_{T} h_{3}=1
\end{aligned}
$$

Furthermore, the topological derivative terms, $D_{T} C_{i j}^{h}$, can be computed with the expression (35) described in Appendix I.

Analogous treatment is performed on equation (14), but it is not shown here.

\subsubsection{Algorithm implementation}

Next, we describe the implementation of the level-set methodology in the context of an FFT technique for computational homogenization. This implementation can 
be compared with alternative approaches of level-set-methodologies reported in the revision paper by van Dijk et al. [9].

The iterative algorithm for solving the topology optimization problems (13) and (14) are described in Appendix II. In particular, two alternative schemes are defined for computing the iterative step length.

\section{i) Parametrization of the level-set-function (LSF)}

The FFT technique makes use of a discretization of the unit cell defined through a uniform grid of $\left(N_{\alpha}+1\right) \times\left(N_{\beta}+1\right) \times\left(N_{\gamma}+1\right)$ points, such as schematized in Figure 6. These points divide the volume in $N_{\alpha} \times N_{\beta} \times N_{\gamma}$ voxels; being $N_{\alpha}, N_{\beta}$ and $N_{\gamma}$ the number of voxels along the coordinate directions $\boldsymbol{g}_{\alpha}, \boldsymbol{g}_{\beta}$ and $\boldsymbol{g}_{\gamma}$, respectively. The vertices of these voxels are the grid points.

The level-set-function $\psi(\boldsymbol{y})$ is defined in the grid points and is identified by $\hat{\psi}_{p}=\psi\left(\boldsymbol{y}_{p}\right)$, where $\boldsymbol{y}_{p}$ is the spatial position of the $p$-th grid point. The set $\left\{\hat{\psi}_{p}\right\}$, for: $p=1, \ldots,\left(N_{\alpha}+1\right)\left(N_{\beta}+1\right)\left(N_{\gamma}+1\right)$ are the level-set-function parameters.
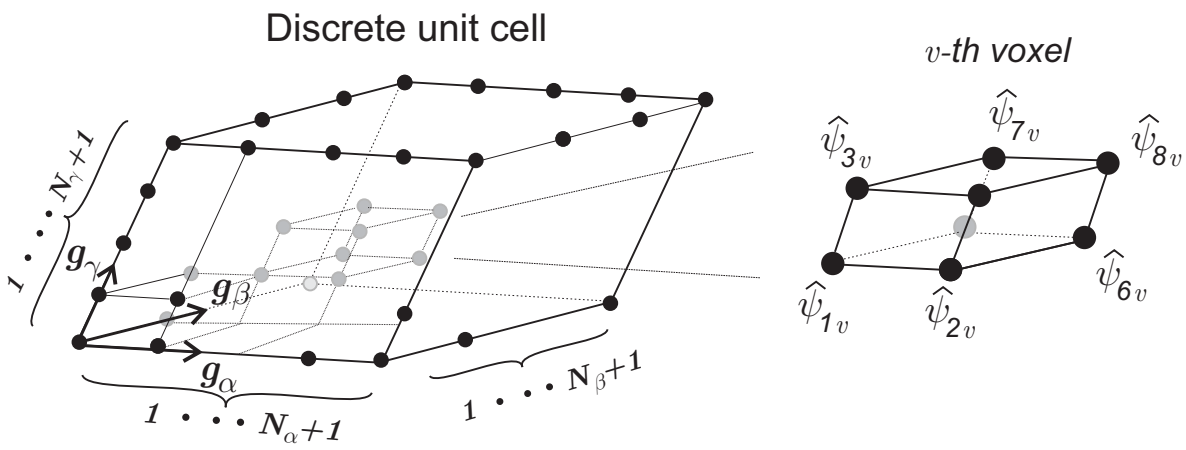

Figure 6: Discrete unit cell. Parameters of the level-set-function associated with the $v$-th voxel.

\section{ii) Geometry mapping from the LSF parametrization}

The elastic property of the $v$-th voxel represented in Figure 6, is characterized with the elasticity tensor $\hat{\boldsymbol{C}}^{v}$ that is computed with the convex combination of the elasticity tensors $\boldsymbol{C}_{M_{1}}$ and $\boldsymbol{C}_{M_{2}}$, of phases $M_{1}$ and $M_{2}$ respectively, as follows:

$$
\hat{\boldsymbol{C}}^{v}=\hat{\omega}^{v} \boldsymbol{C}_{M_{1}}+\left(1-\hat{\omega}^{v}\right) \boldsymbol{C}_{M_{2}}
$$

where the coefficient $\hat{\omega}^{v}$, of the $v$-th voxel, is evaluated with the eight level-set function parameters, $\hat{\psi}_{p_{v}}$ for: $p_{v}=1, \ldots, 8$, that correspond to the $v$-th voxel 
vertices, as follows

$$
\hat{\omega}^{v}=\frac{\sum_{p_{v}=1}^{8}\left|\hat{\psi}_{p_{v}}^{-}\right|}{\sum_{p_{v}=1}^{8}\left|\hat{\psi}_{p_{v}}\right|} \quad ; \quad \hat{\psi}_{p_{v}}^{-}=\left\{\begin{array}{cc}
\hat{\psi}_{p_{v}} & \text { if: } \hat{\psi}_{p_{v}}<0 \\
0 & \text { otherwise }
\end{array}\right.
$$

with $\hat{\omega}^{v} \in[0,1]$. The numerator of this expression is the addition of the absolute values of the level-set function parameters with negative values.

The term $\hat{\omega}^{v}$ plays the role of a smoothed characteristic function, similar to $\chi$ defined in equation (6). According to equations (20) and (21), if the eight level-set-function parameters of a voxel are negative (positive), the elasticity tensor coincides with the elasticity tensor of phase $M_{1}\left(M_{2}\right)$. Alternatively, if the level-set function parameters are positive and negative, the material elastic stiffness can be interpreted as a mixture with proportional stiffness to $\hat{\omega}^{v}$. The salient feature of equation (21) is that a smooth transition from $\boldsymbol{C}_{M_{1}}$ to $\boldsymbol{C}_{M_{2}}$ is induced by a continuous movement of the interface, i.e., the zero iso-surface level-set function, across the voxel.

\section{iii) Updating the LSF parameters}

Once the topological derivative of $\mathcal{T}$ is known, the level-set-function is updated, at iteration $k+1$, as follows:

$$
\psi^{k+1}=\psi^{k}+\tau g
$$

where $g(\boldsymbol{y})$ is defined by:

$$
g(\boldsymbol{y})= \begin{cases}-\left(D_{T} \mathcal{T}\right) & \text { if }: \psi<0 \\ +\left(D_{T} \mathcal{T}\right) & \text { if }: \psi>0\end{cases}
$$

and can be interpreted as a descent direction of $\mathcal{T}$, and $\tau$ is a step length. Additional discussions for determining the value of the $\tau$ are presented in Appendix II.

For the problem (13), the topological derivative of $\mathcal{T}$ is initially computed in the $N_{\alpha} \times N_{\beta} \times N_{\gamma}$ voxels according to equations (18), (19) and (35). Then, they are mapped to the grid points by averaging the values of the neighboring voxels. The resulting nodal derivative at the generic grid point $p$ is denoted $\hat{g}_{p}$. Finally, the level-set function parameters in the grid points are updated with equation (22).

The iterative updating of the Lagrange multipliers $\lambda_{i}$ of the Lagrangian function $\mathcal{T}$ are further discussed in the Appendix II. 


\section{iv) Regularization and filtering of the LSF parameters}

The level-set function parameters are filtered through a discrete convolution product

$$
\psi^{f}\left(\boldsymbol{y}_{p}\right)=(\psi * K)\left(\boldsymbol{y}_{p}\right) ;
$$

where $\psi^{f}$ are the level-set function parameters after the filtering and the discrete convolution product is defined as

$$
\psi^{f}\left(\boldsymbol{y}_{p}\right)=(\psi * K)\left(\boldsymbol{y}_{p}\right)=\sum_{k=1}^{\left(N_{\alpha}+1\right) \times\left(N_{\beta}+1\right) \times\left(N_{\gamma}+1\right)} \hat{\psi}_{k} K\left(\boldsymbol{y}_{k}-\boldsymbol{y}_{p}\right) ;
$$

and the Kernel $K$ is

$$
K(\boldsymbol{x})= \begin{cases}\beta-\frac{\|\boldsymbol{x}\|}{\ell_{f}} & \text { if }:\|\boldsymbol{x}\|<\ell_{f} \\ 0 & \text { if }:\|\boldsymbol{x}\|>\ell_{f}\end{cases}
$$

with the coefficient $\beta$ being taken such that $\sum_{k} K\left(\boldsymbol{y}_{k}-\boldsymbol{y}_{p}\right)=1$, for all $p$. The filter radius $\ell_{f}$ determines the minimum allowed length scale of the designed topology. The topological derivative is also filtered with a similar expression.

\section{v) Convergence criterion}

Convergence is achieved when the following two criteria are accomplished:

a) Following to Amstutz [25], a local optimality criterion of problem (15) is given by the condition

$$
D_{T} \mathcal{T}>0 \quad ; \quad \forall \boldsymbol{y} \in \Omega_{\mu}
$$

which can be implemented by verifying the inequality

$$
\frac{\hat{\boldsymbol{g}} \cdot \hat{\boldsymbol{\psi}}}{\|\hat{\boldsymbol{g}}\|\|\hat{\boldsymbol{\psi}}\|}>\left(1-t o l_{\psi}\right)
$$

where the vectors $\hat{\boldsymbol{g}}$ and $\hat{\boldsymbol{\psi}}$ collect the values of $\hat{g}$ and $\hat{\psi}$, respectively, of the grid points $\left(\hat{\boldsymbol{g}}=\left\{\hat{g}_{p}\right\}\right.$ and $\hat{\boldsymbol{\psi}}=\left\{\hat{\psi}_{p}\right\}$, with $\left.p=1, \ldots,\left(N_{\alpha}+1\right) \times\left(N_{\beta}+1\right) \times\left(N_{\gamma}+1\right)\right)$.

b) All constraints must be satisfied with given prescribed tolerances:

$$
\left|h_{i}\right|<\operatorname{tol}_{h_{i}} \quad ; \quad \text { with } i=1,2,3
$$

\section{vi) Nested grid refinements}

The optimization problems are solved using a grid refinement scheme. Initially, they are solved with a rather coarse grid of points. Once their solutions attain 
the convergence criteria in this coarse grid, the solutions are projected onto a finer grid, and the iterative scheme is restarted.

Each refinement step consists of exactly doubling the number of voxels per cell side, and therefore, from one step to the next, the number of voxels increases with a factor 8 . Using this approach, the level-set function parameters of the coarser grid are projected onto the finer one. The projection procedure is the following: i) For coincident points of both grids, the parameters are identically copied. ii) For those points of the finer grid not coinciding with the points of the coarser grid, the parameters are the average values of the parameters defined in the closest points of the coarser grid, such as it is sketched for a $2 \mathrm{D}$ problem in Figure 7.

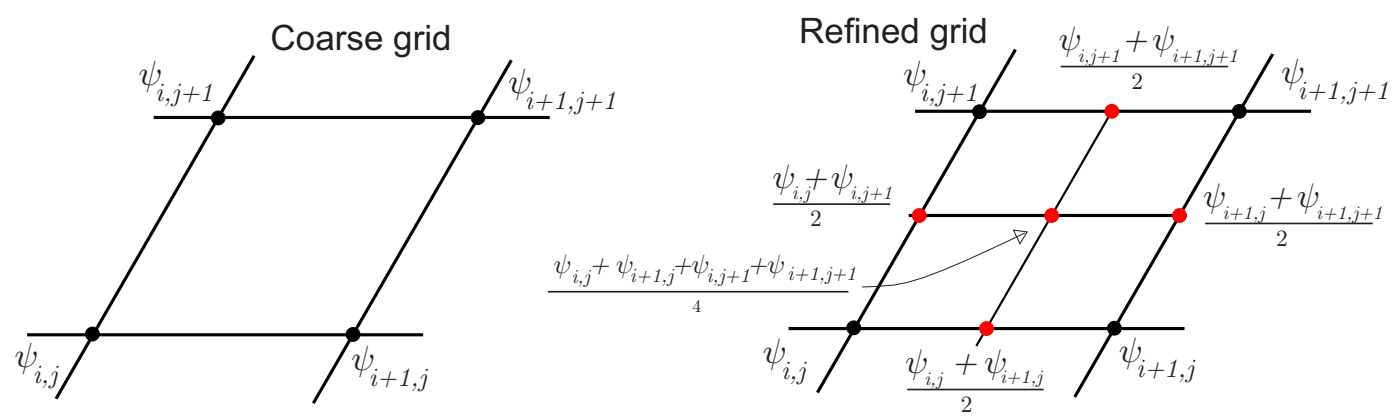

Figure 7: Nested grid refinement procedure. Mapping of the level-set function parameters, from the coarser grid onto the finer one.

\subsection{Strategy for imposing the space group symmetry}

The topology computed at each iteration of the optimization algorithm is forced to exhibit the crystal symmetry defined by a preestablished space group. To get this objective, we adopt the following strategy.

a) First, the set of points belonging to the asymmetric unit ${ }^{4}$ of the design domain is identified.

b) The image points, related to the asymmetric unit points through the symmetry operations of the space group, are next identified. Both set of points depend on the crystal space group.

c) In each iteration of the optimization algorithm, the average values of the levelset function parameters and topological derivatives of the asymmetric unit

\footnotetext{
${ }^{4}$ The asymmetric unit and the corresponding image points are defined for all the space groups in the International Tables of Crystallography ([14]).
} 


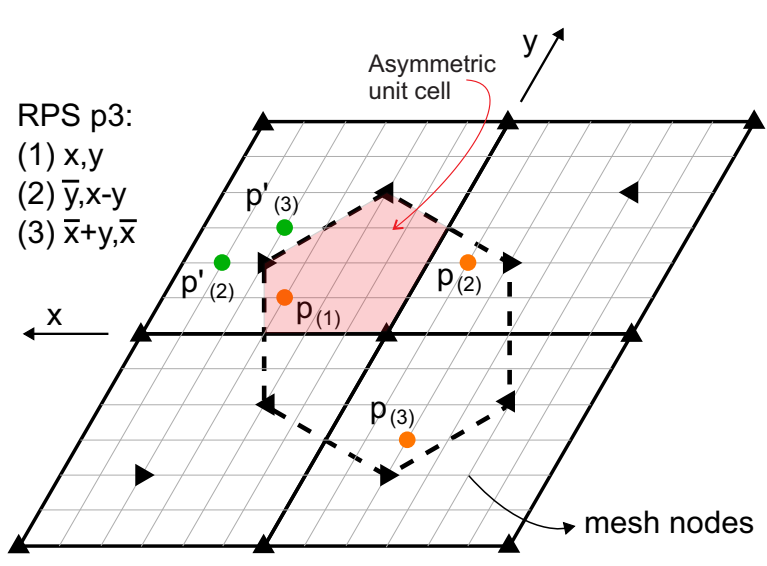

(a)

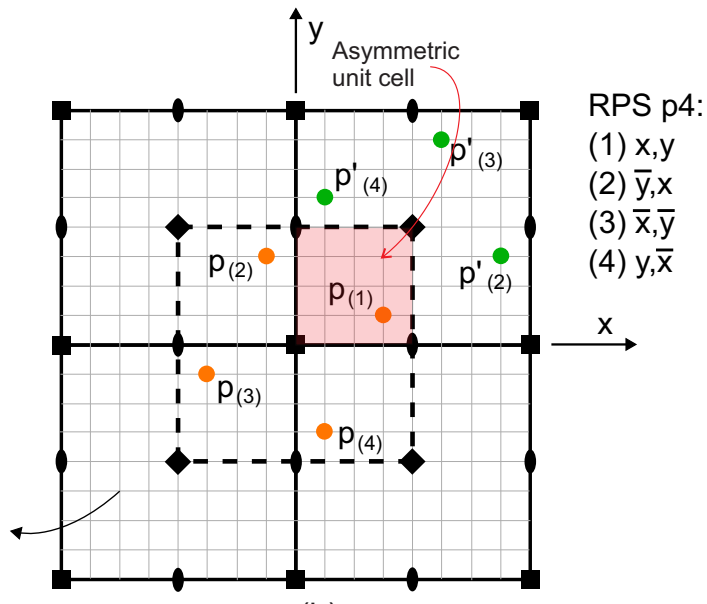

(b)

Figure 8: Procedure to impose the 2D crystal symmetry. Plane groups (a) p3 and (b) $p 4$. Primitive cells are depicted with solid lines and Wigner-Seitz (Voronoi) cells with dotted lines. Orange dots are image points which are located using the RPS information for every point in the asymmetric unit cell. Green dots are the result of the symmetry translations of orange points that lay outside the parallelepiped cell.

point and its image points are computed. These average values are assigned to all of them.

For regular grids of points, such as those used in the FFT scheme, the identification of the image points associated with every point in the asymmetric unit is achieved by using the regular point system $(\mathrm{RPS})^{5}$, see Vainshtein [15]. Examples of RPS and the mechanism of locating the image points in the grid are shown in Figure 8 for two 2D cases. Information about the adopted origin of the coordinate system and the RPS for plane and space groups are specified in the International Tables for Crystallography [14]. In the case of the plane group $p 3$, the coordinates taken as examples of generic coordinates are $x=3 / 6$ and $y=1 / 6$, while for the plane group $p 4$, these coordinates are $x=3 / 8$ and $y=1 / 8 .{ }^{6}$

\footnotetext{
${ }^{5} \mathrm{An}$ RPS is the set of points that are associated with one generic point, occupying an arbitrary spatial position, through the symmetry group operations. The coordinates of these points are expressed as a function of the generic point coordinates.

${ }^{6}$ To be consistent with the notation used in the International Tables of Crystallography ([14]), the coordinates in Figure 8 are denoted $(x, y)$ instead of the usual notation for the contravariant coordinates that we have adopted in this work.
} 


\section{Numerical assessments}

Microarchitecture design problems to attain composites with isotropic elastic properties close to the theoretical estimated bounds are next solved. Main results of this task have been presented by the authors elsewhere, see Yera et al. [2], where the influence of imposing crystal symmetries to attain the desired objective has been evaluated by testing four space groups of the cubic crystal systems for 3D problems. Additional results of similar problems have been reported by Andreassen and Lazarov [26].

The numerical performance of the topology optimization algorithms is analyzed in three specific design cases. They aim at attaining microstructures whose effective properties display: a) one of the most negative Poisson's ratios (auxetic composite); b) the stiffest response and c) pentamodal features. The effective elastic properties of these composites in the space $(\hat{\kappa}, \hat{G})$ occupy the locations displayed in Figure 5. The composites with negative Poisson's ratios have their properties on the left region of the HS-bound. They are composites demanding high shear modulus and small bulk modulus. The stiffest composite is positioned at the upper right bound in the $(\hat{\kappa}, \hat{G})$ space, demanding high $\hat{\kappa}$ and $\hat{G}$ moduli, and pentamode composites are characterized by small shear modulus and are positioned at the lower HS-bound in the same space. Three instances of pentamode composites are solved.

The attained optimal solutions depend on the crystal symmetry adopted for solving the topology optimization problem. Yera et al. have shown that closer solutions to the theoretical bounds can be found by choosing suitable crystal space groups for each case. For example, the space groups I23 and P23 are appropriate to attain maximal properties in the region close to the HS-left bound, coincident with the response of auxetic materials. Furthermore, the proper selection of the optimization domain, $\Omega_{\mu}$, has also a notable influence. In the same paper, Yera et al. also show that topologies with bcc primitive cell provide topologies with closer properties to the HS upper bound than those provided by a simple cubic cell.

\subsection{Specific technique for solving the present numerical as- sessments}

The topology optimization algorithm described in Appendix II, with scheme 2, has been used for solving the auxetic composite. The remaining microarchitectures reported in this Section have been obtained with the same algorithm but using scheme 1.

The optimization process starts by taking a grid with $32 \times 32 \times 32$ voxels. It is followed by posterior refinements till reaching grids of $256 \times 256 \times 256$ voxels $^{7}$. The

\footnotetext{
${ }^{7}$ The sequence of grid refinements consists of taking $32,64,128$ and 256 voxels per spatial dimension.
} 
initial topology configurations in all cases are a random distribution satisfying the target volume fraction of the stiff phase.

The solutions of the problems reported in this work are obtained with the filter radius fixed to zero. However, as it can be observed in the attained topologies of Figures 10 to 14, they do not display a finer sub-scale. This outcome may be due to the adopted grid refinement strategy. The coarse grid taken for starting the optimization process precludes the generation of a fine topology structure. We have observed that in the posterior grid refinement steps, the algorithm does not longer introduce a sub-scale.

\subsection{D topology design problems}

The designed composites have the same elastic properties, for both phases, defined in the previous sub-Section 3.1. Therefore, the Figure 9 copies the HS-bounds in the space $(\hat{\kappa}, \hat{G})$ depicted in Figure 5 . In the same Figure 9, we also show four solutions $\mathrm{A} ; \mathrm{B}, \mathrm{C}$, and $\mathrm{D}$ that have been obtained with the present methodology. They are discussed in sub-Sections 4.2.1, 4.2.2, 4.2.3:case-a and 4.2.3:case-b, respectively. Following the suggestions raised by Yera et al., different space groups have been imposed on the topology optimization problems to get these solutions. The results reported by these authors using grids of 100 voxels are also shown in the Figure.

Finally, the last example in sub-Section 4.2.3:case-c, describes a particular solution of a pentamode composite with a smaller volume fraction $f_{1}=0.1$.

\subsubsection{Auxetic materials}

The synthesis of auxetics materials has recently raised an enormous interest in the community due to the unusual properties that they display, such as increased resistance to indentation, synclasticity, improvement of fracture toughness, etc. (Saxena et al. [27] and Cabras and Brun [28]). For example, Podestá et al. [17] have shown that optimal design of elastic structures demands the use of auxetics materials.

In this test, the objective is to attain an isotropic composite with minimum Poisson's ratio for a relatively high target shear modulus. The problem formulation (14) is used in this case with a target shear modulus ${ }^{8} G^{t g}=0.03$.

The designed microarchitecture has been solved with an imposed space group I23 of the cubic crystal system. Figure 10 displays the resulting topology, and their effective elastic properties are plotted in Figure 9, point A. The components of the attained homogenized elasticity tensor are: $C_{11}^{h}=C_{22}^{h}=C_{33}^{h}=0.041500$,

\footnotetext{
${ }^{8}$ It is worth to mention that solutions reported by Yera et al. [2] and Andreassen and Lazarov [26] show that it is possible to attain more negative Poisson's ratios using smaller target values of the shear modulus $G^{t g}$.
} 


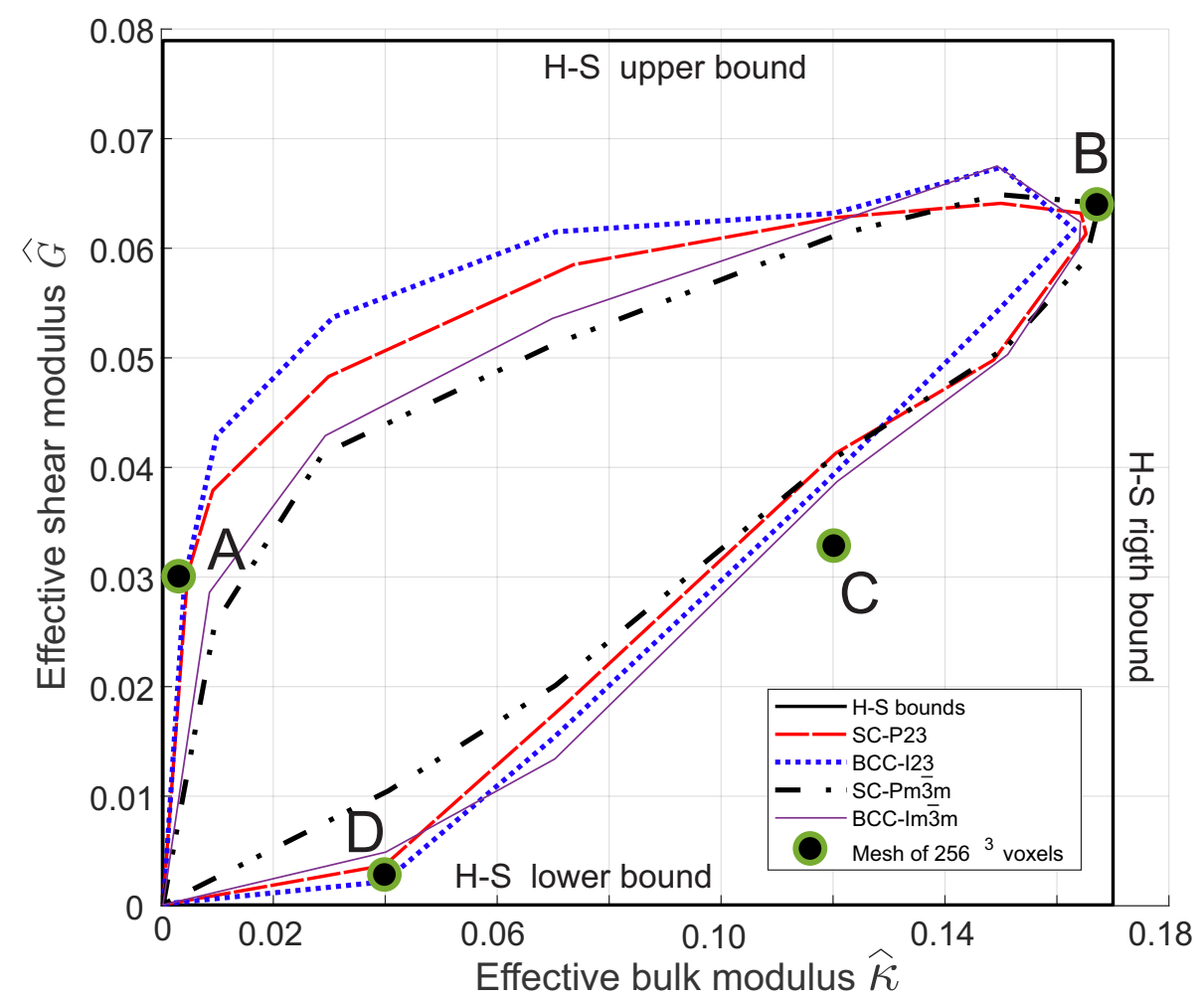

Figure 9: The points A, B, C and D in the plane $(\hat{\kappa}, \hat{G})$ have been obtained with the present methodology and grids of $256 \times 256 \times 256$ voxels. The curves have been taken from Yera et al. [2] with the space groups and unit cells of the cubic crystal system: P23 with a primitive cubic cell (SC-P23), I23 with a bcc primitive cell (BCCI23), $P m \overline{3} m$ with primitive cubic cell $(\mathrm{SC}-P m \overline{3} m)$ and $I m \overline{3} m$ with bcc primitive cell (BCC-Im $\overline{3} m)$. HS-bounds of effective properties for two-phase isotropic 3D composites according to Hashin and Shtrikman [22] (HS-bounds). 


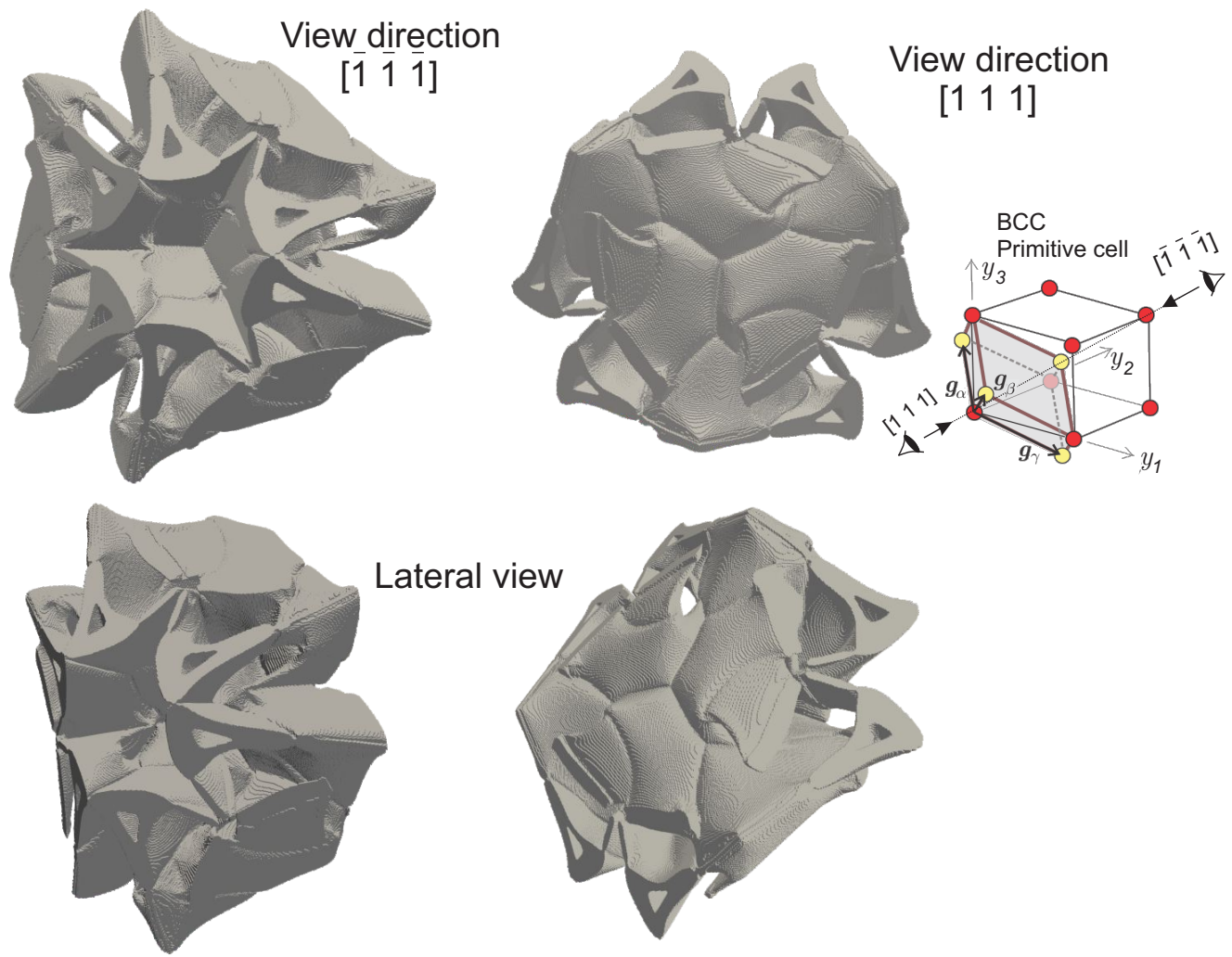

Figure 10: Primitive cell of the composite with elastic properties corresponding to Point A in Fig 9. Imposed space group I23. Primitive cell observed from two

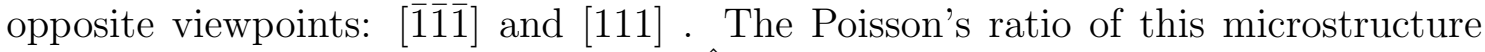
is -0.82 , the effective shear modulus $\hat{G}=0.0301$ and the effective bulk modulus $\hat{\kappa}=0.0013405$.

$C_{12}^{h}=C_{23}^{h}=C_{13}^{h}=-0.018739$ and $C_{44}^{h}=C_{55}^{h}=C_{66}^{h}=0.060176$. Thus, the attained Poisson's ratio is -0.82 and the volume fraction is $f_{1}=0.3383$.

We use the Zener ratio to quantify the anisotropy of the effective material properties having cubic symmetry. The Zener ratio is computed with the formula: $\xi=C_{44}^{h} /\left(C_{11}^{h}-C_{12}^{h}\right)$. A value 1 for this ratio indicates that $\boldsymbol{C}^{h}$ is isotropic.

The present solution has a Zener ratio of value $\xi=0.9989$, very close to 1 , and therefore, it satisfies almost exact isotropic response. It is important to remark that several auxetic composites reported in the literature are strongly anisotropic.

\section{Deformation mechanism of the designed auxetic material}

Additional insight respect to the deformation mechanism characterizing the microarchitecture of Figure 10 can be conceived by analyzing an alternative, but equivalent, solution. First, notice that the topology depicted in Figure 10, obtained with 
the space group I23, tends to show a higher symmetry than the enforced one. According to this observation, we achieve a simpler microstructure, though slightly less efficient, by imposing the space group $I \overline{4} 3 \mathrm{~m}$. The so-attained microstructure is described in Figure 11 and its effective Poisson's ratio is -0.74. The homogenized elasticity tensor components of the microarchitecture is: $C_{11}^{h}=C_{22}^{h}=C_{33}^{h}=0.042101$, $C_{12}^{h}=C_{23}^{h}=C_{13}^{h}=-0.017933$ and $C_{44}^{h}=C_{55}^{h}=C_{66}^{h}=0.0600$. The Zener ratio is $\xi=0.9994$. Notice that the main difference between the solutions of Figures 10 and 11 lies on the thin sub-structures that are commonly attained when extreme materials are designed.

The deformation mechanism of the simpler microstructure in Figure 11 is next explained. Figures 11 (a) and (b) show the primitive and conventional cells. Figures $11(\mathrm{~d})$ and (e) present the details of the interior region of the conventional cell and the external faces of the conventional cell, respectively. Also, they sketch the deformation mechanism when the microstructure is subjected to a macroscopic strain.

Three cell configurations are presented in Figures 11 (d) and (e). The central pictures display the original configurations of the cell. The pictures on the right side display the deformed cell when it is stretched in the direction [ 1000$]$. The pictures on the left side display the deformed cell when it is compressed in the same direction. Notice the original and deformed positions of the points 1,2 and 3 in the three configurations of Figure 11 (d). It can be seen the microstructure unfolding mechanism that is activated when it is stretched; the points are moving away and the dihedral angle $\alpha$ increases. Alternatively, in the reverse direction, in a compressive regime, the microstructure folding mechanism is activated; the points are approaching and the dihedral angle $\alpha$ decreases. The points 1, 2 in the three configurations of the Figure 11 (e) display a similar folding/unfolding mechanism.

We point out that the deformation mechanism of the microstructure could be envisaged through the simpler deformation mechanism displayed by the origami in Figure (c). Both structures deform following a similar pattern.

\subsubsection{Maximum stiffness materials}

With the present approach, we have noted that the topology optimization algorithm finds solutions close to the HS-right bound more easily than solutions close to the HSupper bound. According to this observation, in this problem, we find the topology by solving a maximization problem with the formulation (13). The target bulk modulus is $\kappa^{t g}=0.167$ close to the upper bulk modulus, $\kappa^{u}=0.1704$, estimated by the HS-bounds. The enforced space group is $P m \overline{3} m$. The design domain is the primitive cell of the simple cubic Bravais lattice.

Figure 12 displays the attained topology. It is constituted of a void volume covered with a layered structure in three orthogonal directions that contributes to 

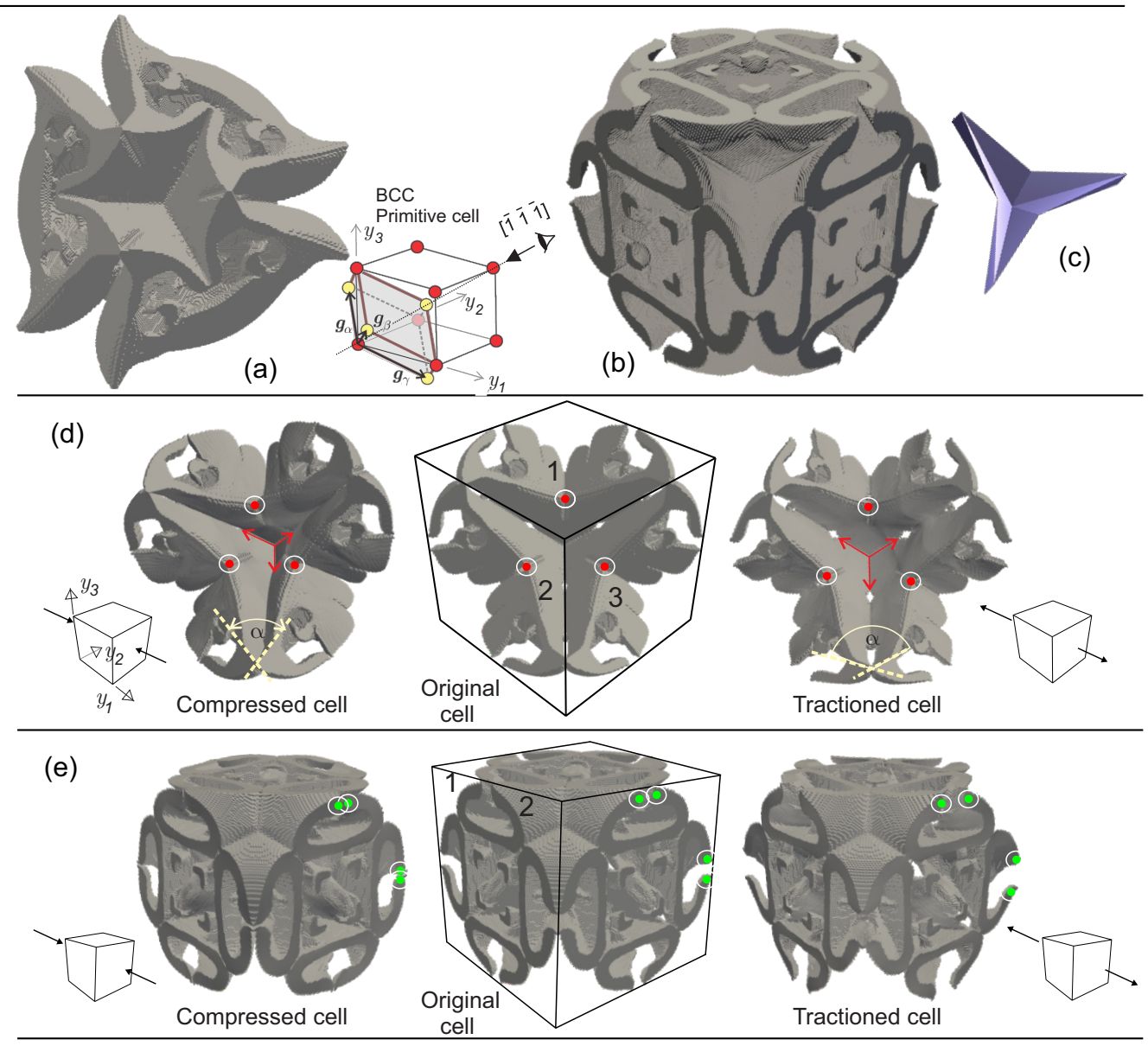

Figure 11: Auxetic composite with space group $I \overline{4} 3 \mathrm{~m}$ and Poisson's ratio -0.74 . (a) Primitive cell observed from viewpoint $[\overline{1} \overline{1} \overline{1}]$. (b) Conventional cell. (c) Origami displaying a similar deformation mechanism to the auxetic composite. (d) Half of the conventional cell constituted by the contribution of four primitive cells. Internal region views. (e) External views of the conventional cell. 

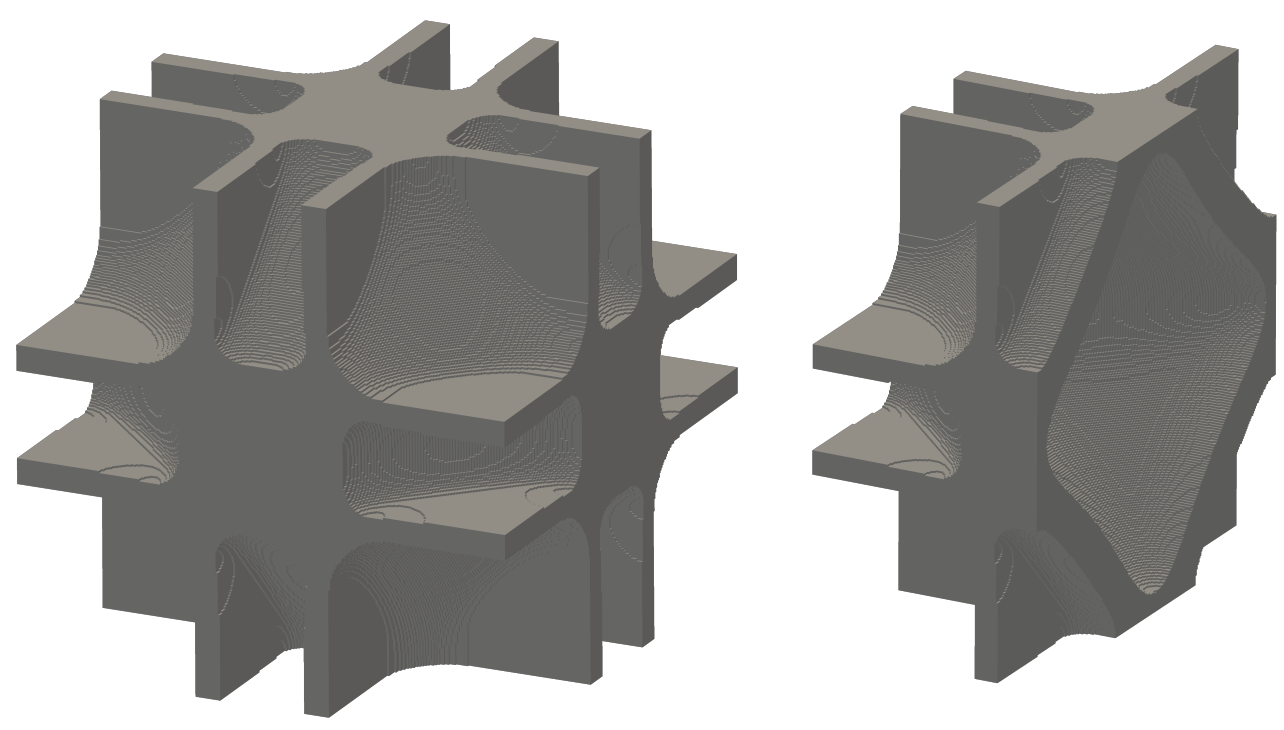

Figure 12: Topology of the maximum stiffness composite, point B in Figure 9, designed with a $P m \overline{3} m$ space group. (a) Primitive cell. (b) Trimmed microcell showing the interior void.

increasing the shear stiffness. This topology can be compared with the microstructure of Figure 14 obtained for high bulk modulus and low shear stiffness.

The homogenized elasticity tensor components of the microarchitecture displayed in Figure 12 is: $C_{11}^{h}=C_{22}^{h}=C_{33}^{h}=0.252523, C_{12}^{h}=C_{23}^{h}=C_{13}^{h}=0.124672$ and $C_{44}^{h}=C_{55}^{h}=C_{66}^{h}=0.127826$, with effective bulk and shear moduli plotted in Figure 9, point B. The Zener ratio is $\xi=0.9998$.

\subsubsection{Pentamode materials}

The name of pentamode materials has been coined by Milton and Cherkaev $[10]^{9}$ to designate the class of composites displaying a stiff response for one characteristic strain mode while displaying easy modes of deformations for the remaining five characteristic strain modes. We consider that the characteristic strain modes are assumed to be the six eigenvectors of the elasticity tensor. Thus, for a typical isotropic material, the stiff deformation mode can be associated with the eigenvalue coinciding with the bulk modulus, while the compliant response is associated with the shear modulus. It means that a pentamode material displays an almost null shear modulus jointly with a non-null bulk modulus. Pentamode materials have an interest because any achievable effective elastic material can be generated using an

\footnotetext{
${ }^{9}$ At the same time, Sigmund [29] has independently introduced this kind of composites in the context of inverse homogenization problems.
} 
assemblage of pentamode materials, such as mentioned in the paper by Milton and Cherkaev [10]. In this reference work, the authors also propose a natural candidate for the pentamode materials, being a diamond-type structure in which four linkages meet at a point.

\section{Case a: microstructure of a pentamode composite attained with $P 23$ space group (point D in Figure 9)}

We solve the problem (13) with $\kappa^{t g}=0.04$ and the imposed space group P23. The solved microstructure is displayed in Figure 13, and it has effective properties which correspond to the point D in Figure 9. The components of its homogenized elasticity tensor are: $C_{11}^{h}=C_{22}^{h}=C_{33}^{h}=0.04363, C_{12}^{h}=C_{13}^{h}=C_{23}^{h}=0.03817$ and $C_{44}^{h}=C_{55}^{h}=C_{66}^{h}=0.00630$. Thus, the Zener coefficient is $\xi=1.1538$, with a ratio: $\kappa^{h} / G^{h} \approx 13$. The six eigenvalues of the elasticity tensor are 0.1997, 0.0630, 0.0630, $0.0630,0.0547$ and 0.00547 , respectively, Then, the ratios between the maximum $\lambda_{\text {max }}$, the medium $\lambda_{\text {med }}$ or the minimum $\lambda_{\text {min }}$ eigenvalues are: $\lambda_{\text {max }} / \lambda_{\text {med }}=19$ and $\lambda_{\max } \lambda_{\min }=22$,

The topology is constituted of four interconnected helix-like sub-structures. Each sub-structure is formed by three linked bulky elements. Details of the links are displayed in Figure 13-b. Unlike the Milton microstructure that is analyzed in the following case-c, in this topology, the linkage of the three bulky elements is not reduced to a point. Additionally, as it can be observed in Figure 13-a, the internal region of the cell displays the four helix-like sub-structures which are interconnected through a small microstructure constituted by four bars.

From this result, we can conclude that the deformation mechanisms generated by the four helix-like linked substructures are responsible for providing an almost isotropic effective elastic response of the composite, while their linkage would probably need the introduction of a sub-topology, with a smaller length scale to further decrease the effective shear modulus.

\section{Case b: Microstructure of a pentamode composite attained with the $\operatorname{Im} \overline{3} m$ space group (point $\mathbf{C}$ in Figure 9)}

Although the microstructure of Figure 13 displays a high bulk to shear moduli ratio, it is attained by imposing a low target bulk modulus $\kappa^{t g}<\kappa^{u} / 4$. With higher values of $\kappa^{t g}$, the ratio $\kappa^{h} / G^{h}$ of the attained composites decreases and their material properties tend to move away from the lower theoretical HS-bound.

A typical case is the topology shown in Figure 14, which corresponds to Point $\mathrm{C}$ in Figure 9, obtained with the formulation (13), with $\kappa^{t g}=0.12$ and imposing a space group symmetry $\operatorname{Im} \overline{3} m$. This result shows two well-recognized sub-structures which are linked through a bar-like system of elements. The components of the homogenized elasticity tensor of this microstructure are: $C_{11}^{h}=C_{22}^{h}=C_{33}^{h}=0.16458$, 

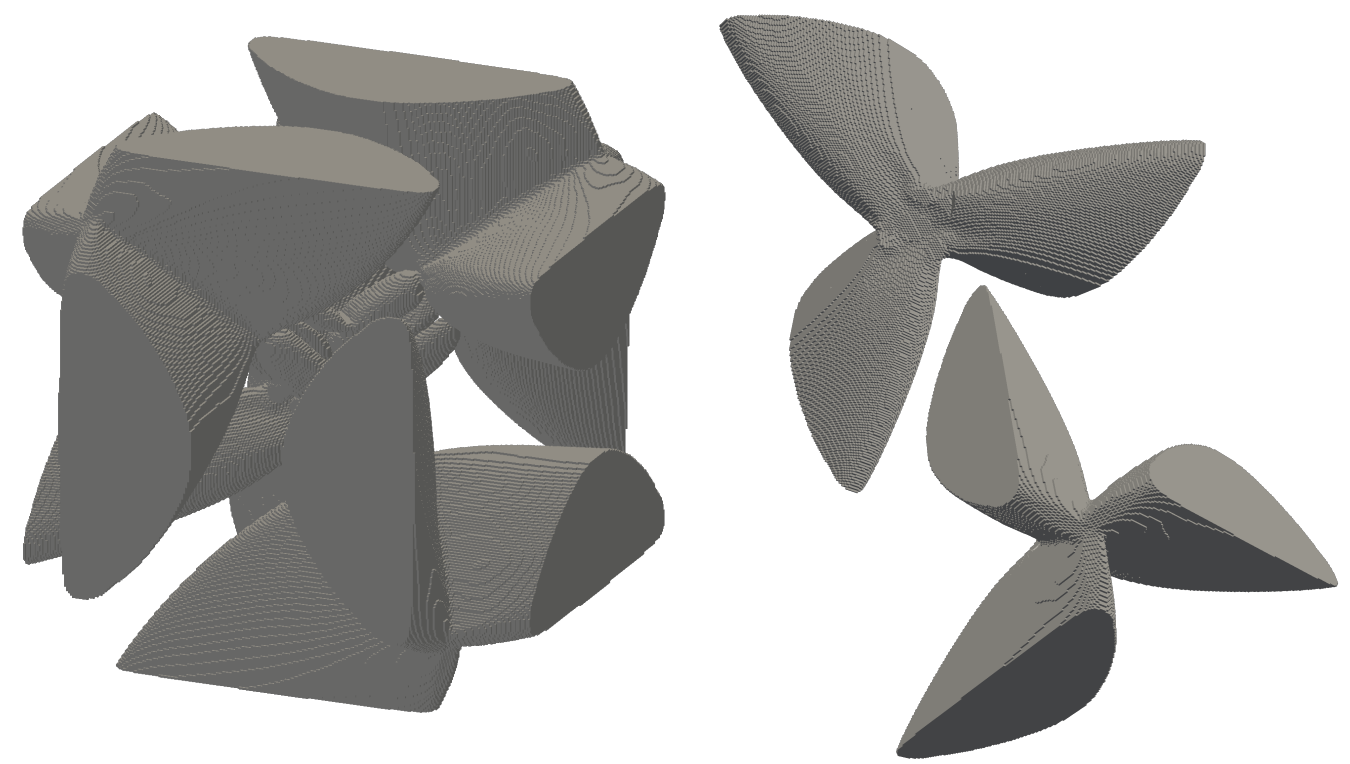

Figure 13: Pentamode material attained with the space group P23. Effective properties corresponds to point D of Figure 9. (a) Primitive cell; (b) detail of the joints of the substructures.

$C_{12}^{h}=C_{13}^{h}=C_{23}^{h}=0.09815$ and $C_{44}^{h}=C_{55}^{h}=C_{66}^{h}=0.06500$. Thus, the Zener coefficient is $\xi=0.9785$,

The attained shear modulus of this microstructure is far from the lower HSbound. However it is interesting to note certain similitude with the topologies reported by Sigmund [4] for the equivalent problem, identified as the Walpole point problem in 2D. Both 2D and 3D structures have the maximum possible symmetry, i.e. $p 6 m m$ with a hexagonal lattice in the $2 \mathrm{D}$ case, and $I m \overline{3} m$ with a bcc lattice in the $3 \mathrm{D}$ case. Another remarkable aspect is that both topologies tend to include additional sub-structures displaying a smaller length scale. Compare Point 9 of Figure 7 in Sigmund's work and the details in Figure 14-(b). In the 2D case, the lower length scale structure is a laminated material.

In the 3D case reported here, two independent saddle-shaped sub-structures conform, after cell assembly, a system of hollow "tubes" which are interconnected by parallel bars/cylinders in three directions, such as observed in the details depicted in Figure 14. Remarkably, Milton and Camar-Eddine [30] have recently discussed similar kinds of microstructures in the context of isotropic pentamode material. The microstructures proposed by these authors have almost zero shear modulus and can be realized with a system of parallel cylinders, having characteristic sizes much lower than the microarchitecture size, which are inserted into structures having maximum bulk and shear moduli. These cylinders are placed in a sufficient number of orientations to ensure the isotropy of the composite. Additionally, in the discussion 

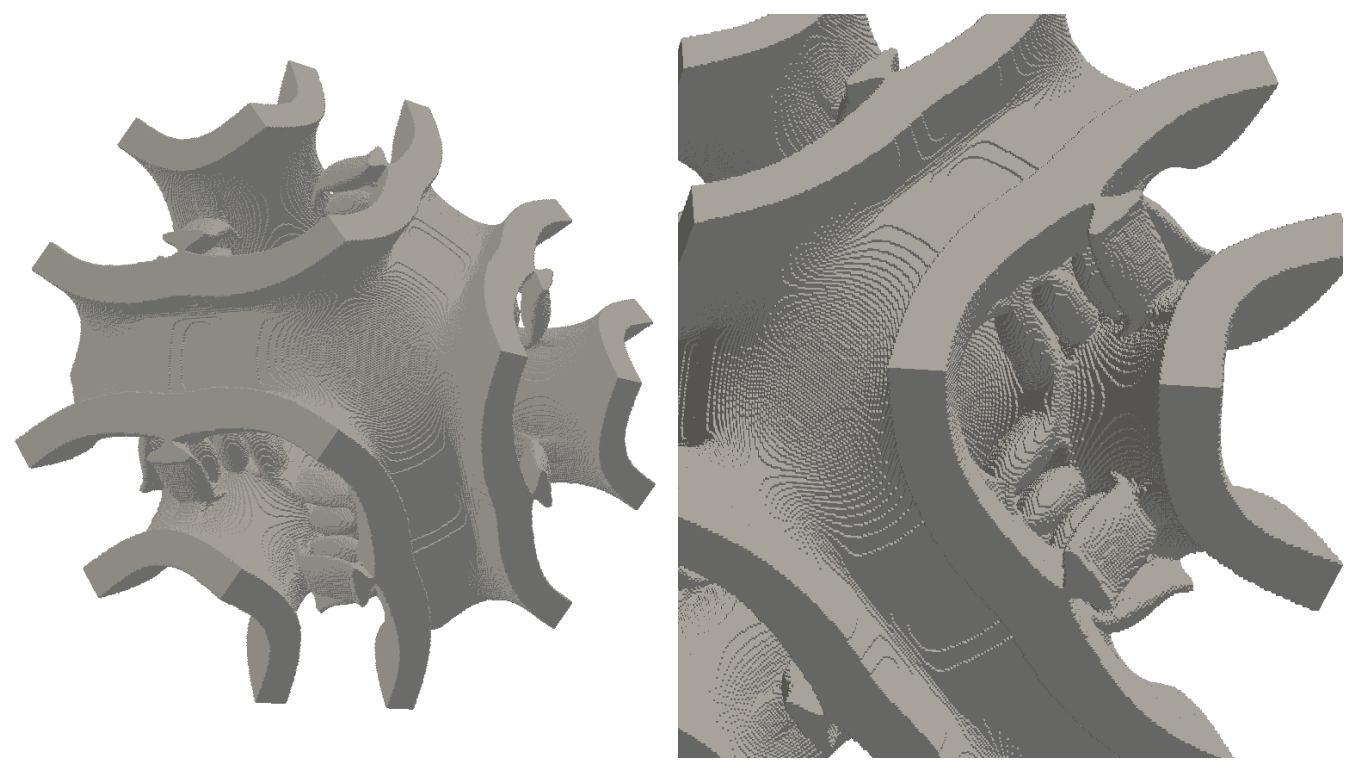

Figure 14: Topology obtained with the $I m \overline{3} m$. Effective properties corresponds to Point $\mathrm{C}$ in Fig 9. Primitive cell and details of links between the two sub-structures constituting the composite.

presented by Milton et al. [31], it has been pointed out that multiscale substructures are necessary to achieve simple topologies with properties close to the theoretical bounds. According to these comments and due to the design constraints that we adopt in this work, the bar system observed in Figure 14 does not constitute a true lower scale length of the microstructure, and therefore, they could be the reason for preventing an additional decrement of the shear modulus.

\section{Case c: microstructure of a pentamode composite attained with $F \overline{4} 3 m$ space group and smaller volume fraction}

The diamond's cubic structure depicted in Figure 15, with the internal atoms $i$ equal to the face atoms $f$ of the conventional cell, has a crystal symmetry with the $F d \overline{3} m$ space group and fcc Bravais lattice. When the atoms $i$ are different to atoms $f$, the crystal symmetry is lowered to the space group $F \overline{4} 3 m$.

An interesting point demonstrating the potentiality of the present methodology lies in the fact that by imposing a space group $F \overline{4} 3 m$, with fcc Bravais lattice, we attain a microarchitecture similar to that proposed by Milton et al., displaying the higher symmetry $F d \overline{3} m$ of the diamond-like structure. This solution is shown in Figure 16 and has been obtained for a stiff volume fraction of 0.1. This microstructure is slightly different from that reported by Kadic et al. [32]. In the present case, the optimal bars conforming the diamond-like microstructure are similar to ellipsoids, 


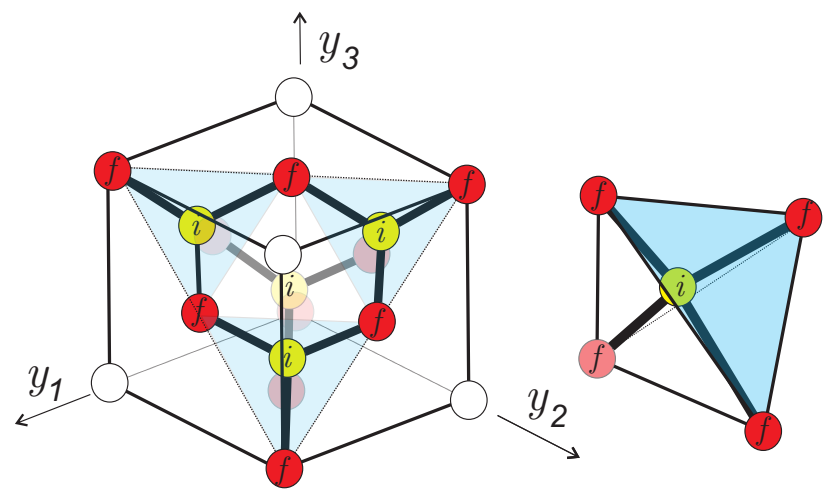

Figure 15: Diamond's crystal structure. Atoms $f$ are on the unit cell faces and atoms $i$ are on the interior of the cell.

with the section of the bars not being exactly circular but similar to hexagons with rounded vertices, as can be noted in the Figure.

A detail of the geometry of the bar joints are shown in Figure 16-(b).

The homogenized elasticity tensor is $C_{11}=0.00994, C_{12}=0.00936$ and $C_{44}=$ 0.00157. The six eigenvalues of this tensor are: $0.02866,0.00157,0.00157,0.00157$, 0.00057 , and 0.00057 . Thus, the six eigenvalues of the elasticity tensor are 0.02866 , $0.00157,0.00157,0.00157,0.00057$, and 0.00057 , respectively. And the ratios between the maximum, $\lambda_{\text {max }}$, the medium, $\lambda_{\text {med }}$ or the minimum, $\lambda_{\text {min }}$, eigenvalues are: $\lambda_{\text {max }} / \lambda_{\text {med }}=18$ and $\lambda_{\text {max }} / \lambda_{\text {min }}=50$. The Zener coefficient is $\xi>2$ resulting a composite whose properties are far from being isotropic. In this case, the specific tolerance of the algorithm corresponding to the isotropy constraint has been handled to force the convergence conditions.

The ratio $\kappa^{h} / G^{h}=16$ is low if compared with the results reported by Kadic et al. However, note that the solution of Kadic et al. corresponds to a volume fraction less than 0.05 .

\subsection{Computational burden for solving the topology opti- mization problems}

Several additional tests have been performed to assess the computational burden demanded by the present methodology. The four topology optimization problems denoted A, B, C and D in Figure 9 have been solved with:

i) two space groups: P23 and I23, and

ii) using the iterative schemes 1 and 2 described in Appendix II.

Ten instances of each problem have been tested. For each instance, the iterative process is initiated with a random distribution of the stiff phase and a grid of $32 \times$ 

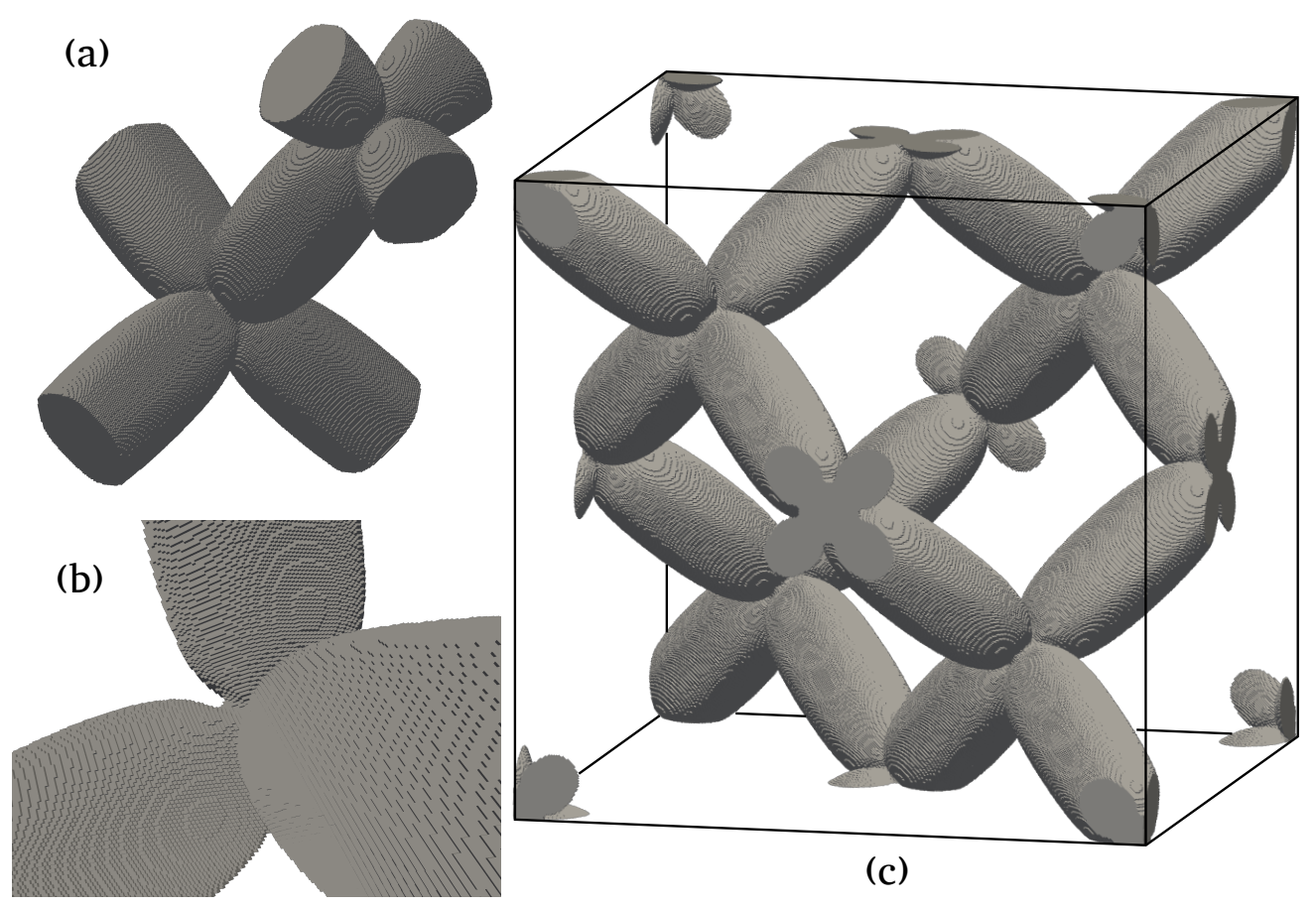

Figure 16: Topology obtained with space group $F \overline{4} 3 m$. (a) Primitive parallelepiped cell; (b) Detail of the joints shape; (c) Conventional cell.

$32 \times 32$ voxels. After convergence, a new iterative process is started with a grid of $64 \times 64 \times 64$ voxels. In this case, the initial material configuration is the projection of the coarse grid solution onto the fine grid.

The results, in terms of computational burden, are reported in Table 2. This table compares the number of tests that have converged, the total number of iterations required to converge, the number of homogenization evaluations that have been performed and the attained best objective function value of each problem.

The Table rows denoted: $C / T$ report the ratio between the number of converged tests divided by the total numbers of tested instances, which is 10 in all cases; $M N I$ report the number of iterations needed to converge (the mean value of the converged instances); $M N H$ report the number of times (average) that the homogenization elasticity tensor has been computed; and $B O f$ report the attained optimum value of the objective function.

The Table columns P23 and I23 report the results of the five problems with topologies satisfying the crystal symmetries with either the $P 23$ or $I 23$ space groups; Grid $32^{3}$ and Grid $64^{3}$ identify the results obtained with either $32 \times 32 \times 32$ or $64 \times 64 \times 64$ voxels; and Sch 1 and $S c h 2$ identify the iterative scheme, described in Appendix II, adopted to solve the problem. The scheme using a predefined step length (parameter $\tau$ in equation (22)) is denoted with Sch 1. The line search scheme 
Table 2: Computational burden for solving the topology design problems A, B, C and D of Figure 9. First column identifies the problem.

\begin{tabular}{|c|c|c|c|c|c|c|c|c|c|}
\hline & & \multicolumn{4}{|c|}{ P23 } & \multicolumn{4}{|c|}{$\mathrm{I} 23$} \\
\hline & & \multicolumn{2}{|c|}{ Grid $32^{3}$} & \multicolumn{2}{|c|}{ Grid $64^{3}$} & \multicolumn{2}{|c|}{ Grid $32^{3}$} & \multicolumn{2}{|c|}{ Grid $64^{3}$} \\
\hline & & Sch 1 & Sch 2 & Sch 1 & Sch 2 & Sch 1 & Sch 2 & Sch 1 & Sch 2 \\
\hline \multirow{4}{*}{ A } & $\mathrm{C} / \mathrm{T}$ & $6 / 10$ & $10 / 10$ & $5 / 6$ & $\overline{10 / 10}$ & $10 / 10$ & $10 / 10$ & $10 / 10$ & $\overline{10 / 10}$ \\
\hline & MNI & 843 & 366 & 193 & 114 & 816 & 264 & 94 & 87 \\
\hline & $\overline{\mathrm{MNH}}$ & 845 & 1120 & 194 & 447 & 818 & 872 & 95 & 305 \\
\hline & BOf & $5.94 \mathrm{e}-3$ & $6.67 \mathrm{e}-3$ & $3.67 \mathrm{e}-3$ & $3.74 \mathrm{e}-3$ & $4.98 \mathrm{e}-3$ & $5.30 \mathrm{e}-3$ & $3.18 \mathrm{e}-3$ & $2.44 \mathrm{e}-3$ \\
\hline \multirow{4}{*}{ B } & $\mathrm{C} / \mathrm{T}$ & $9 / 10$ & $10 / 10$ & $9 / 9$ & $10 / 10$ & $5 / 10$ & $9 / 10$ & $4 / 8$ & $8 / 9$ \\
\hline & MNI & 409 & 607 & 68 & 78 & 702 & 906 & 127 & 198 \\
\hline & MNH & 411 & 2162 & 69 & 221 & 704 & 2752 & 128 & 457 \\
\hline & BOf & $6.67 \mathrm{e}-2$ & $6.58 \mathrm{e}-2$ & $6.69 \mathrm{e}-2$ & $6.79 \mathrm{e}-2$ & $6.66 \mathrm{e}-2$ & $6.39 \mathrm{e}-2$ & $6.66 \mathrm{e}-2$ & $6.54 \mathrm{e}-2$ \\
\hline \multirow{4}{*}{$\mathrm{C}$} & $\mathrm{C} / \mathrm{T}$ & $10 / 10$ & $10 / 10$ & $10 / 10$ & $8 / 10$ & $9 / 10$ & $6 / 10$ & $9 / 9$ & $6 / 6$ \\
\hline & MNI & 973 & 629 & 166 & 118 & 875 & 309 & 88 & 58 \\
\hline & $\mathrm{MNH}$ & 975 & 1522 & 167 & 385 & 877 & 915 & 89 & 167 \\
\hline & BOf & $3.51 \mathrm{e}-2$ & $3.72 \mathrm{e}-2$ & $3.40 \mathrm{e}-2$ & $3.50 \mathrm{e}-2$ & $3.91 \mathrm{e}-2$ & $3.97 \mathrm{e}-2$ & $3.75 \mathrm{e}-2$ & $3.83 \mathrm{e}-2$ \\
\hline \multirow{4}{*}{$\mathrm{D}$} & $\mathrm{C} / \mathrm{T}$ & $5 / 10$ & $8 / 10$ & $4 / 5$ & $8 / 8$ & $7 / 10$ & $8 / 10$ & $7 / 7 / 7$ & $7 / 8$ \\
\hline & $\mathrm{MNH}$ & 859 & 736 & 23 & 36 & 929 & 630 & 228 & 107 \\
\hline & MNH & 861 & 2060 & 24 & 116 & 931 & 1754 & 229 & 317 \\
\hline & BOf & $3.62 \mathrm{e}-3$ & $3.34 \mathrm{e}-3$ & $2.92 \mathrm{e}-3$ & $2.50 \mathrm{e}-3$ & $5.58 \mathrm{e}-3$ & $3.14 \mathrm{e}-3$ & $3.99 \mathrm{e}-3$ & $2.61 \mathrm{e}-3$ \\
\hline
\end{tabular}

is denoted with Sch 2 .

According to the data reported in Table 2, we observe that the number of iteration required for convergence notably changes according to the problem. Additionally, we can estimate the robustness performance of the algorithm by analyzing the number of converged solutions respect to the total number of performed tests.

In most tested cases, scheme 2 demands more homogenization evaluations than scheme 1 to achieve the solution, resulting in a more costly procedure. However, scheme 2 does not need to guess the step length for generic cases, which yields a more reliable procedure.

\section{Conclusions}

In this paper, we have shown that the microarchitecture design of elastic materials attained through the solution of topology optimization problems, combined with the imposition of pre-defined crystal symmetries, is a viable and robust methodology for 
designing 3D metamaterials with effective elastic properties close to the theoretical bounds.

To reach this objective, we have adopted two similar topology optimization problems that are formulated as inverse homogenization techniques. The implementation details of the algorithm for solving these formulations have also been presented. The algorithm is based on a level-set function technique with the sensitivities, for the iterative correction of the level-set function, evaluated through the topology derivative of the problem.

We have used an FFT technique reported in the literature to compute efficiently the homogenized elastic properties of 3D composites. The contrast factor for modeling the void phase has been taken small enough to provide accurate solutions without substantially penalizing the computational cost required by the FFT technique. A good performance of the FFT technique has been attained after introducing grey voxels in the interfaces which simulate materials with intermediate properties of the constituent phases.

To confirm the computational efficiency of this homogenization technique, we have presented a detailed numerical assessment consisting of computing the homogenized elasticity tensor of a periodic microstructure with an fcc Bravais lattice. Results, in terms of computational time vs. accuracy, have been compared with solutions obtained using the FEM. Under these circumstances and including the grey material in the model, the FFT technique has a favorable performance if compared with the FEM.

The design technique, as well as the computation of the homogenized elastic properties of composites with arbitrary crystal symmetries and periodic topologies, requires the use of primitive cells of Bravais lattices that are associated with the pre-defined space group imposed to the topology. One of the most salient features of these cells is that they are not necessarily rectangular prisms. Therefore, the use of the FFT technique reported in the literature has to be adapted to these situations where the cell faces are non-orthogonal. This issue has specifically been addressed in this work.

To attain the microarchitectures with effective properties close to the theoretical bounds, we have imposed crystal symmetries with different space groups which have been adopted in concordance with the conclusions of a previous paper of the authors (Yera et al. [2]). The imposed space groups are compatible with the cubic crystal system. Therefore, the obtained topologies have effective elastic properties with cubic symmetry. Based on this evidence, the isotropy of these microstructures can be measured with the Zener coefficient who is reported for all cases discussed in the paper. Excluding the pentamode material of case $\mathrm{c}$ in sub-Section 4.2.3, in all the remaining cases, the Zener coefficients are close to 1 . Therefore, we conclude that the isotropic response is fulfilled in almost the complete set of designed topologies.

In sub-Section 4.3, we have evaluated the computational burden required for 
solving several topology design cases. An important conclusion can be drawn from the data reported in Table 2 . It refers to the relatively high number of tests that have reached convergence. We conclude that the methodology with the crystal symmetry ingredients displays good and robust performances.

An interesting final remark about the integrated design strategy, here discussed, is its suitability for imposing different space group symmetries due to the regular grids used for solving the FFT scheme.

\section{Acknowledgments}

The authors acknowledge the financial support from CONICET and ANPCyT (grants PIP 2013-2015 631 and PICT 2014-3372 and 2016-2673).

We thank Dr. Ricardo Lebensohn, from Los Alamos National Laboratory, for the discussion hold about some topics related to the FFT homogenization technique in parallelepiped domains addressed in this work.

\section{APPENDIX I: Homogenized elasticity tensor and its topological Derivative}

Let us consider the problem sketched in Figure 1. The macro-stress $\boldsymbol{\sigma}$ and macrostrain $\boldsymbol{E}$ are the volumetric average, in the micro-cell $\Omega_{\mu}$, of the corresponding micro-stress $\boldsymbol{\sigma}_{\mu}$ and micro-strain $\boldsymbol{\varepsilon}_{\mu}$ observed at the micro-scale: $\boldsymbol{\sigma}=\left\langle\boldsymbol{\sigma}_{\mu}\right\rangle$ and $\boldsymbol{E}=\left\langle\boldsymbol{\varepsilon}_{\mu}\right\rangle$. Also, $\boldsymbol{\varepsilon}_{\mu}(\boldsymbol{y})$ can be split into the average strain $\boldsymbol{E}$ plus a fluctuation term $\tilde{\varepsilon}_{\mu}(\boldsymbol{y})$ with zero average value in $\Omega_{\mu}$ :

$$
\varepsilon_{\mu}(\boldsymbol{y})=\boldsymbol{E}+\tilde{\varepsilon}_{\mu}(\boldsymbol{y})
$$

The fluctuation micro-strain is the symmetric gradient of a fluctuation displacement field, $\tilde{\boldsymbol{u}}$ which is periodic on the boundary $\Gamma_{\mu}$ of $\Omega_{\mu}$. The displacement fluctuations $\tilde{\boldsymbol{u}}$ are periodic along the directions defined by the primitive vectors of the Bravais lattice.

Stresses and strains at the macro-scale are related through the homogenized elasticity tensor $\boldsymbol{C}^{h}$ :

$$
\boldsymbol{\sigma}=\boldsymbol{C}^{h} \boldsymbol{E}
$$

and stresses and strains at the micro-scale are related through the constitutive law:

$$
\boldsymbol{\sigma}_{\mu}(\boldsymbol{y})=\boldsymbol{C}(\boldsymbol{y}) \varepsilon_{\mu}(\boldsymbol{y})
$$

where $\boldsymbol{C}(\boldsymbol{y})$ is the elasticity tensor of phase at the point $\boldsymbol{y}$. Additionally, $\boldsymbol{\sigma}_{\mu}$ is a self-equilibrated stress field: $\nabla \cdot \boldsymbol{\sigma}_{\mu}=\mathbf{0}$ in $\Omega_{\mu}$.

By following to Moulinec et al, the micro-scale field equations with the corresponding boundary conditions can be rewritten as an auxiliary problem and reduced 
to the Lippmann-Schwinger integral equation for $\tilde{\boldsymbol{\varepsilon}}_{\mu}(\tilde{\boldsymbol{u}})$, which, finally, can be iteratively solved in the frequency space, using a fixed-point algorithm.

\section{Homogenized elasticity tensor}

Let us define the canonical base of the macro-strain tensors $\mathcal{E}:=\left\{\boldsymbol{E}^{I_{1}}, \ldots, \boldsymbol{E}^{I_{6}}\right\}$, where the indices $I_{1}, I_{2}, \ldots, I_{6}$ identify every one of the six strain bases $\boldsymbol{E}^{I_{m}}$. The six components of each strain base is written in the conventional way, $E_{i j}^{I_{n}}$, with $i, j=1,2,3$. Then, the components of the canonical strain bases are defined by

$$
\boldsymbol{E}_{i j}^{I_{n}}= \begin{cases}1 & \text { if }(i, j) \in I_{n} \\ 0 & \text { if }(i, j) \notin I_{n}\end{cases}
$$

where the following association between component indices, $i, j$, and strain base indices, $I_{n}$, is defined: $(1,1) \in I_{1} ;(2,2) \in I_{2} ;(3,3) \in I_{3} ;\{(1,2),(2,1)\} \in I_{4}$; $\{(2,3),(3,2)\} \in I_{5} ;\{(1,3),(3,1)\} \in I_{6}$.

The components of the homogenized elasticity matrix ${ }^{10}$ is computed with the expression:

$$
\boldsymbol{C}_{m n}^{h}=\boldsymbol{\sigma}\left(\boldsymbol{E}^{I_{m}}\right): \boldsymbol{E}^{I_{n}} \quad ; \quad m, n=1,2, \ldots, 6 ;
$$

where $\boldsymbol{\sigma}\left(\boldsymbol{E}^{I_{m}}\right)$ is the homogenized macro-stress satisfying the multiscale equations, defined above, for the given macro-strain $\boldsymbol{E}^{I_{m}}$.

Thus, six problems $\boldsymbol{\sigma}\left(\boldsymbol{E}^{I_{m}}\right)$, with $m=1, \ldots 6$, are solved. Associated to each one of them, the micro-strain $\varepsilon_{\mu}^{I_{m}}$ and the micro-stress $\boldsymbol{\sigma}_{\mu}^{I_{m}}$ are also computed in the micro-cell. According to equation (29), the micro-strain $\varepsilon_{\mu}^{I_{m}}$ is split as follows:

$$
\varepsilon_{\mu}^{I_{m}}(\boldsymbol{y})=\boldsymbol{E}^{I_{m}}+\tilde{\varepsilon}_{\mu}^{I_{m}}(\boldsymbol{y})
$$

where $\tilde{\varepsilon}_{\mu}^{I_{m}}$ is a fluctuation term with zero average value on the micro-cell.

\section{Topological derivative of the homogenized elasticity tensor}

The book by Novotny and Sokołowski [23] describes the topological derivative of the energy function associated with an elastic boundary value problem defined in a given spatial domain. Following this approach, Giusti [33] develops the topological derivative of the effective elasticity tensor obtained using a computational homogenization procedure for 2D problems. This term has subsequently been used by Amstutz et al. [7] for solving a topology optimization algorithm. Here, we only present a summary of these concepts and the expression used for the topology derivative in 3D problems, see Giusti [34] and Lopes et al. [24].

The topological derivative of the homogenized elasticity tensor represents the sensitivity of this tensor to infinitesimal spherical perturbation in the homogenization domain, such as sketched in Figure 17. Let us consider that, at the point $\hat{\boldsymbol{y}}$ of

\footnotetext{
${ }^{10}$ At this point, we are implicitly introducing the Kelvin notation for tensors.
} 


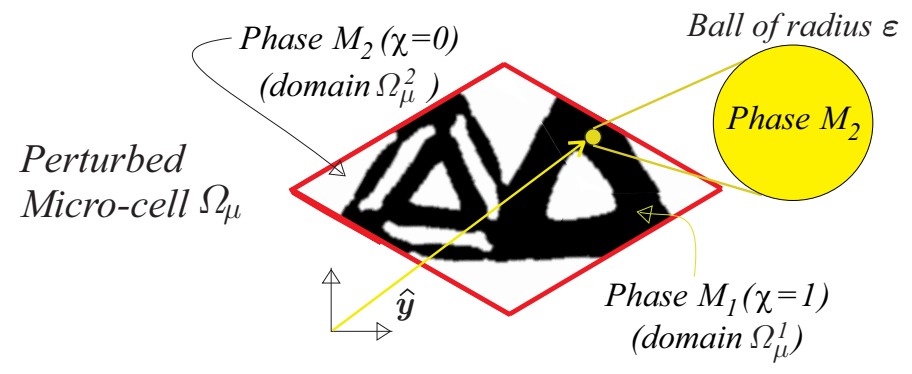

Figure 17: Perturbed micro-cell domain.

the microcell, it exists the base elastic material with the Lamè parameters $(\lambda ; \mu)$ and Poisson's ratio $\nu$. This material is substituted by an infinitesimal spherical perturbation with another elastic material with parameters $\left(\lambda_{0} ; \mu_{0}\right)$. Then, the topological derivative of the homogenized elasticity tensor can be computed with the expression:

$$
D_{T} \boldsymbol{C}_{m n}^{h}(\chi(\hat{\boldsymbol{y}}))=\boldsymbol{\varepsilon}_{\mu}^{I_{m}}(\hat{\boldsymbol{y}}): \mathbb{P}: \boldsymbol{\varepsilon}_{\mu}^{I_{n}}(\hat{\boldsymbol{y}}) \quad ; \quad m, n=1, \ldots, 6
$$

where $\mathbb{P}$ is the polarization tensor ${ }^{11}$.

$$
\mathbb{P}=\alpha_{1}\left(\alpha_{2}(\mathbf{1} \otimes \mathbf{1})+2 \mathbb{I}\right)
$$

the symbols 1 and $\mathbb{I}$ represent the second and fourth order unit tensor, respectively, and the coefficient $\alpha_{1}$ and $\alpha_{2}$ are:

$$
\begin{aligned}
\alpha_{1} & =\left(\frac{15 \mu \delta_{\mu}(\nu-1)}{15 \mu(1-\nu)+2 \delta_{\mu}(5 \nu-4)}\right) \\
\alpha_{2} & =\frac{\delta_{\lambda}\left[15 \mu \lambda(1-\nu)+2 \lambda \delta_{\mu}(5 \nu-4)\right]-2 \delta_{\mu}\left(\lambda \delta_{\mu}-5 \mu \nu \delta_{\lambda}\right)}{5 \delta_{\mu}\left[3 \mu \lambda(1-\nu)-3 \mu \nu \delta_{\lambda}-\lambda \delta_{\mu}(1-2 \nu)\right]} ;
\end{aligned}
$$

with $\delta_{\lambda}=\lambda-\lambda_{0} ; \delta_{\mu}=\mu-\mu_{0}$.

The strains $\varepsilon_{\mu}^{I_{m}}$ and $\varepsilon_{\mu}^{I_{n}}$ in equation (35) are the solutions of the FFT technique to each one of the six problems $(m, n=1,2, \ldots, 6)$ and are given by equation (34). Note from (35) that the topological derivative of $\boldsymbol{C}^{h}$ is defined in each point of the micro-cell $\left(\hat{\boldsymbol{y}} \in \Omega_{\mu}\right)$.

\section{APPENDIX II: Iterative schemes for solving the optimization algorithms}

We have implemented two alternative iterative schemes for solving the equations governing the optimization algorithm which are described in sub-Section 3.4. In this Appendix, we describe both implementations.

\footnotetext{
${ }^{11}$ Additional description and properties of the polarization tensor for 3D problems can be found in [35], where it is called the Elastic Moment Tensor (EMT)
} 
Both implementations are inspired by the Augmented Lagrangian technique and adopt a fixed penalty parameter. The first scheme uses a pre-defined linearly decreasing step size, $\tau$. This parameter determine the size the level-set function update equation (22). The second scheme determines the step size, $\tau$, using a linear search technique. The step size of the first scheme and how it is decreased is defined through a previous adjust procedure.

Both schemes are summarized in the following Box.

\section{General Algorithm}

Initialization: Level-set function $\psi^{0}$; Lagrange multipliers $\lambda_{i}^{0}$; penalty parameter $\alpha$; Amstutz criteria tolerance tol $_{\psi}$; constraints tolerances tol $_{h_{i}}$.

Iteration $k+1$ :

1. Build the characteristic function $\chi\left(\psi^{k}\right)$ defined in equation (12).

2. Perform the homogenization.

3. Evaluate the augmented Lagrangian $\mathcal{T}\left(\psi^{k}, \lambda^{k}\right)$ defined in equation (16)

4. Compute the topological derivative $D_{\psi} \boldsymbol{C}_{m n}^{h}(\chi(\hat{\boldsymbol{y}}))$ using equation (35); apply the filter; impose the symmetries.

5. Compute the search direction of the augmented Lagrangian problem, $g^{k}$, with equation (23), and using $D_{\psi} \mathcal{T}\left(\psi^{k}, \lambda^{k}\right)$ from equation (18).

6. Determine the steep size parameter $\tau$.

- scheme 1: Linearly decreased with $k$.

- scheme 2: $\left\{\begin{array}{l}\text { Choose a safewarded minimum step size } \tau_{\text {min }} \text {. } \\ \text { Perform a linear search }: \tau \in\left[\tau_{\text {min }}, 1\right] .\end{array}\right.$

7. Update the level-set function $\psi^{k+1}$ with equation (22); apply the filter; impose the symmetries.

8. Update the Lagrange multipliers: $\lambda_{i}^{k+1}=\lambda_{i}^{k}+\alpha h_{i}$.

9. Convergence test using equations (27) and (28)

The line search procedure for determining the step size, scheme 2 , is based on the Moré and Thuente [36] algorithm. We use the topological derivative in the sense of the usual derivative. 


\section{References}

[1] M. Kadic, G.W. Milton, M. van Hecke, and M. Wegener. 3D metamaterials. Nature Reviews Physics, 1:198-210, 2019.

[2] R. Yera, N. Rossi Cabral, G. Méndez, and A.E Huespe. Topology design of 2D and 3D elastic material microarchitectures with crystal symmetries displaying isotropic properties close to their theoretical limits. In press: Applied Materials Today, 2019. DOI: 10.1016/j.apmt.2019.100456.

[3] O. Sigmund. Materials with prescribed constitutive parameters: an inverse homogenization problem. International Journal of Solids and Structures, 31 (17):2313-2329, 1994.

[4] O. Sigmund. A new class of extremal composites. Journal of the Mechanics and Physics of Solids, 48(2):397-428, 2000.

[5] M.P. Bendsoe and O. Sigmund. Topology optimization: theory, methods, and applications. Springer Science \& Business Media, 2003.

[6] S. Amstutz and H. Andrä. A new algorithm for topology optimization using a level-set method. Journal of Computational Physics, 216(2):573-588, 2006.

[7] S. Amstutz, S.M. Giusti, A.A. Novotny, and E.A. de Souza Neto. Topological derivative for multi-scale linear elasticity models applied to the synthesis of microstructures. International Journal for Numerical Methods in Engineering, 84(6):733-756, 2010.

[8] G. Allaire, F. De Gournay, F. Jouve, and A. Toader. Structural optimization using topological and shape sensitivity via a level set method. Control and cybernetics, 34(1):59, 2005.

[9] N.P. van Dijk, K. Maute, M. Langelaar, and F. Van Keulen. Level-set methods for structural topology optimization: a review. Structural and Multidisciplinary Optimization, 48(3):437-472, 2013.

[10] G.W. Milton and A.V. Cherkaev. Which elasticity tensors are realizable? Journal of engineering materials and technology, 117(4):483-493, 1995.

[11] C.G. Méndez, J.M. Podestá, S. Toro, A.E. Huespe, and J. Oliver. Making use of symmetries in the three-dimensional elastic inverse homogenization problem. Journal for Multiscale Computational Engineering, 17:261-280, 2019.

[12] J.M. Podestá, C.G. Méndez, S. Toro, and A.E. Huespe. Symmetry considerations for topology design in the elastic inverse homogenization problem. Journal of the Mechanics and Physics of Solids, 128:54-78, 2019. 
[13] J.F. Nye. Physical Properties of Crystals: Their representation by tensors and matrices, volume 146. Clarendon Press-Oxford., 2006.

[14] E. Prince; Boston ; London, editor. International Tables for Crystallography. Volume C, Mathematical, Physical and Chemical Tables. Dordrecht, 2004. Published for the International Union of Crystallography by Kluwer Academic Publishers.

[15] B.K. Vainshtein. Fundamentals of crystals: Symmetry, and methods of structural crystallography, volume 1. Springer Science \& Business Media, 2013.

[16] E. Hitzer and C. Perwass. Visualization of fundamental symmetries in nature. In Proceedings of Fuzzy System Symposium (FSS 2009), Tsukuba, Japan, 14-16 Jul., 2009. http://spacegroup.info/.

[17] J.M. Podestá, C.G. Méndez, S. Toro, A.E. Huespe, and J. Oliver. Material design of elastic structures using voronoi cells. International Journal for $\mathrm{Nu}$ merical Methods in Engineering, 115(3):269-292, 2018.

[18] H. Moulinec and P. Suquet. A numerical method for computing the overall response of nonlinear composites with complex microstructure. Computer methods in applied mechanics and engineering, 157(1-2):69-94, 1998.

[19] D.J. Eyre and G.W. Milton. A fast numerical scheme for computing the response of composites using grid refinement. The European Physical JournalApplied Physics, 6(1):41-47, 1999.

[20] I. Cohen. Simple algebraic approximations for the effective elastic moduli of cubic arrays of spheres. Journal of the Mechanics and Physics of Solids, 52(9): 2167-2183, 2004.

[21] J.G. Berryman and G.W. Milton. Microgeometry of random composites and porous media. Journal of Physics D: Applied Physics, 21(1):87, 1988.

[22] Z. Hashin and S. Shtrikman. A variational approach to the theory of the elastic behaviour of multiphase materials. Journal of the Mechanics and Physics of Solids, 11(2):127-140, 1963.

[23] A.A. Novotny and J. Sokołowski. Topological derivatives in shape optimization. Springer Science \& Business Media, 2012.

[24] C.G. Lopes, R.B. dos Santos, and A.A. Novotny. Topological derivative-based topology optimization of structures subject to multiple load-cases. Latin American Journal of Solids and Structures, 12(5):834-860, 2015. 
[25] S. Amstutz. Analysis of a level set method for topology optimization. Optimization Methods and Software, 26(4-5):555-573, 2011.

[26] E. Andreassen and O. Lazarov, B.S.and Sigmund. Design of manufacturable 3D extremal elastic microstructure. Mechanics of Materials, 69(1):1-10, 2014.

[27] K. K. Saxena, R. Das, and E.P. Calius. Three decades of auxetics research- materials with negative poisson's ratio: a review. Advanced Engineering Materials, 18(11):1847-1870, 2016.

[28] L. Cabras and M. Brun. Auxetic two-dimensional lattices with poisson's ratio arbitrarily close to- 1. In Proceedings of the Royal Society of London A: Mathematical, Physical and Engineering Sciences, volume 470, pages 1-23, 2014.

[29] O. Sigmund. Tailoring materials with prescribed elastic properties. Mechanics of Materials, 20(4):351-368, 1995.

[30] G.W. Milton and M. Camar-Eddine. Near optimal pentamodes as a tool for guiding stress while minimizing compliance in 3d-printed materials: A complete solution to the weak g-closure problem for 3d-printed materials. Journal of the Mechanics and Physics of Solids, 114:194-208, 2018.

[31] Graeme Milton, Marc Briane, and Davit Harutyunyan. On the possible effective elasticity tensors of 2-dimensional and 3-dimensional printed materials. Mathematics and Mechanics of Complex Systems, 5(1):41-94, 2017.

[32] M. Kadic, T. Bückmann, N. Stenger, M.l Thiel, and M. Wegener. On the practicability of pentamode mechanical metamaterials. Applied Physics Letters, 100(19):191901, 2012.

[33] S.M. Giusti. Análise de sensibilidade topológica em modelos constitutivos multiescalas. PhD thesis, Laboratório Nacional de Computa cão Científica, LNCC, Brazil, 2009.

[34] S.M. Giusti. Personal communication. 2019.

[35] H. Ammari, P. Calmon, and E. Iakovleva. Direct elastic imaging of a small inclusion. SIAM Journal on Imaging Sciences, 1(2):169-187, 2008.

[36] J.J. Moré and D.J. Thuente. Line search algorithms with guaranteed sufficient decrease. ACM Transactions on Mathematical Software, 20:286-307, 1994. 